\title{
Status of the James Webb Space Telescope Integrated Science Instrument Module System
}

\author{
Matthew A. Greenhouse*, Michael P. Drury, Jamie L. Dunn, Stuart D. Glazer, Ed Greville, \\ Gregory Henegar, Eric L. Johnson, Ray Lundquist, John C. McCloskey, Raymond G. Ohl IV, \\ Robert A. Rashford, and Mark F. Voyton \\ Goddard Space Flight Center, Greenbelt, MD 20771
}

\begin{abstract}
The Integrated Science Instrument Module (ISIM) of the James Webb Space Telescope (JWST) is discussed from a systems perspective with emphasis on development status and advanced technology aspects. The ISIM is one of three elements that comprise the JWST space vehicle and is the science instrument payload of the JWST. The major subsystems of this flight element and their build status are described.
\end{abstract}

Keywords: JWST, Instrumentation

\section{INTRODUCTION}

The James Webb Space Telescope (Figure 1) is under development by NASA for launch during 2014 with major contributions from the European and Canadian Space Agencies. The JWST mission is designed to enable a wide range of science investigations across four broad themes: [1] observation of the first luminous objects after the Big Bang, [2] the evolution of galaxies, [3] the birth of stars and planetary systems, and [4] the formation of planets and the origins of life. ${ }^{1,2,3}$

Its Integrated Science Instrument Module (ISIM) is the science payload of the JWST. ${ }^{4,5}{ }^{2}$ Along with the telescope and spacecraft, the ISIM is one of three elements that comprise the JWST space vehicle. At $1.4 \mathrm{mT}$, it makes up approximately $20 \%$ of both the observatory mass and cost to launch. In order to maximize efficiency with respect to mass, power, and nonrecurring engineering cost, the four JWST science instruments are not designed as traditional stand-alone systems; rather, the ISIM provides nine key subsystems that are shared by each of the science instruments. These are the: [1] optical metering structure, [2] ISIM Electronics Compartment (IEC), [3] harness radiator, [4] ISIM Command and Data Handling System (ICDH), [5] ISIM Remote Services Unit (IRSU), [6] electrical harness system, [7] thermal control system, [8] flight software system, and [9] on-board script system. In the following sections, we briefly describe the science instruments (which are covered in detail elsewhere in this conference) and provide detail on their shared support systems.

* matt.greenhouse@nasa.gov, 301 286-4517 


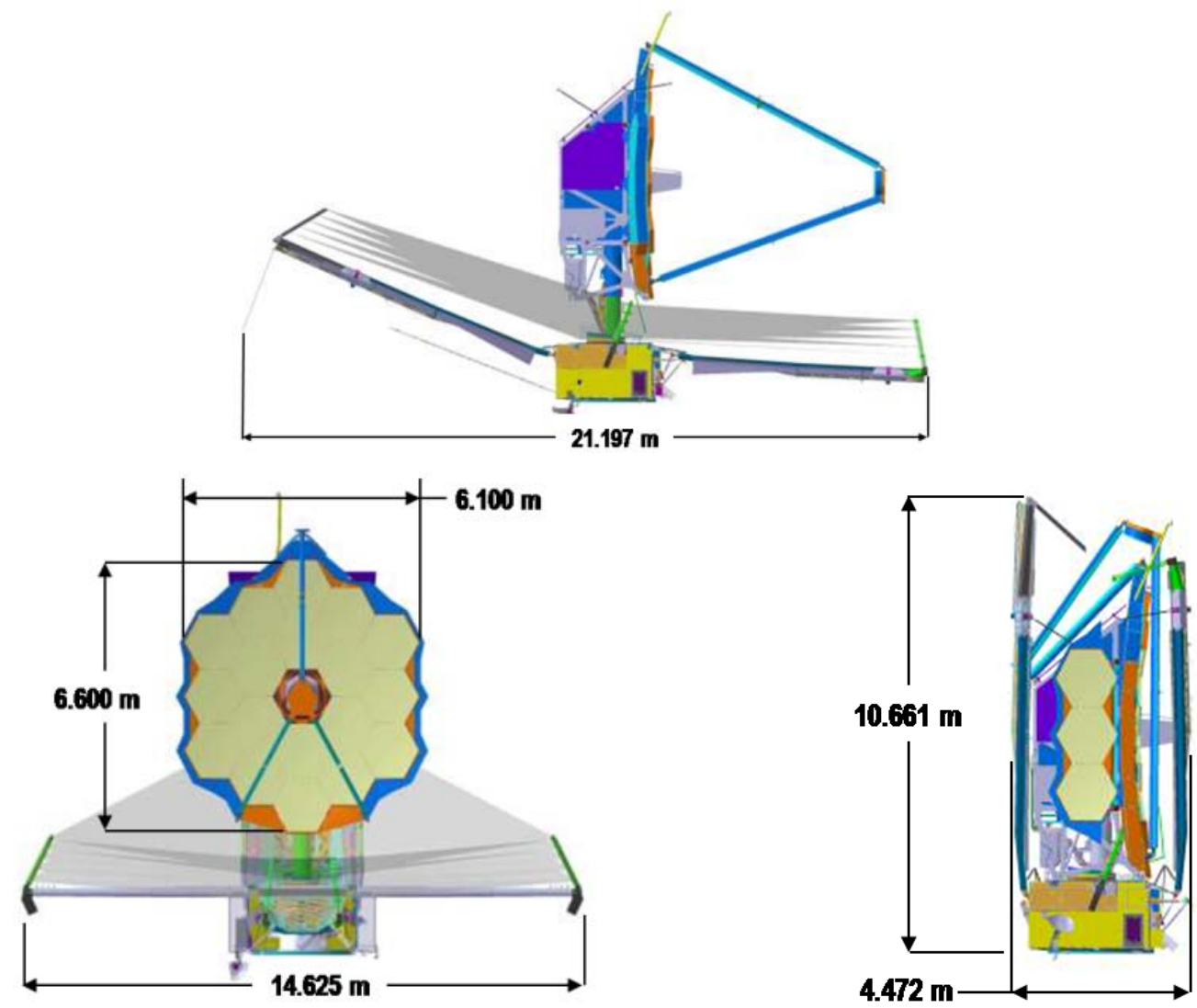

Figure 1: The JWST is shown in its deployed (left and center) and stowed (right) configurations.

\section{SCIENCE INSTRUMENTATION}

\subsection{The Near-Infrared Camera (NIRCam)}

Near-infrared imagery is provided by the NIRCam instrument ${ }^{7,8}$ shown in Figure 2. This camera provides high angular resolution wide-field imagery over the $0.6-5$ micron spectrum. Table 1 summarizes the key elements of NIRCam's capabilities, which remain aimed at providing efficient surveying in two filters simultaneously with a $2 \times 2$ arc minute field. The detector pixel scale is chosen to optimally sample the telescope point spread function across this wavelength range by use of a dichroic beam splitter. Two identical optical modules image adjacent fields of approximately 4 square arcminutes to provide full redundancy for telescope wavefront sensing. The detector arrays for each wavelength regime in NIRCam have sensitivity matched to the needs of the particular channel -- the short wavelength channel uses HgCdTe with a $2.5 \mu \mathrm{m}$ cut-off and the long wavelength arm uses HgCdTe with a $5.1 \mu \mathrm{m}$ cut-off. Using two types of detector material relaxes out-of-band blocking requirements for NIRCam's bandpass filters. Occulting coronagraphy, yielding a rejection ratio of $\sim 10^{4}$, is provided in both long and short wavelength channels. All focal plane arrays support high cadence sub-array exposures to provide a high dynamic range capability for exoplanet transit observations. The instrument is cooled to $\sim 40 \mathrm{~K}$ using passive radiators.

The NIRCam Engineering Test Unit (ETU) and the associated instrument-level test program has been completed (Figure 2) and the ETU has been delivered to GSFC for fit-check integration and other testing with the flight ISIM. The NIRCam also serves as the wave front sensor for figure control of the JWST primary mirror and includes pupil plane optics (dispersed Hartman sensors, a grism, and defocus lenses) and deployable pupil imaging optics for this 
purpose. The NIRCam is being developed by the University of Arizona with Lockheed Martin Advanced Technology Center. Further detail on the NIRCam is presented in paper 7731-11 of this conference.

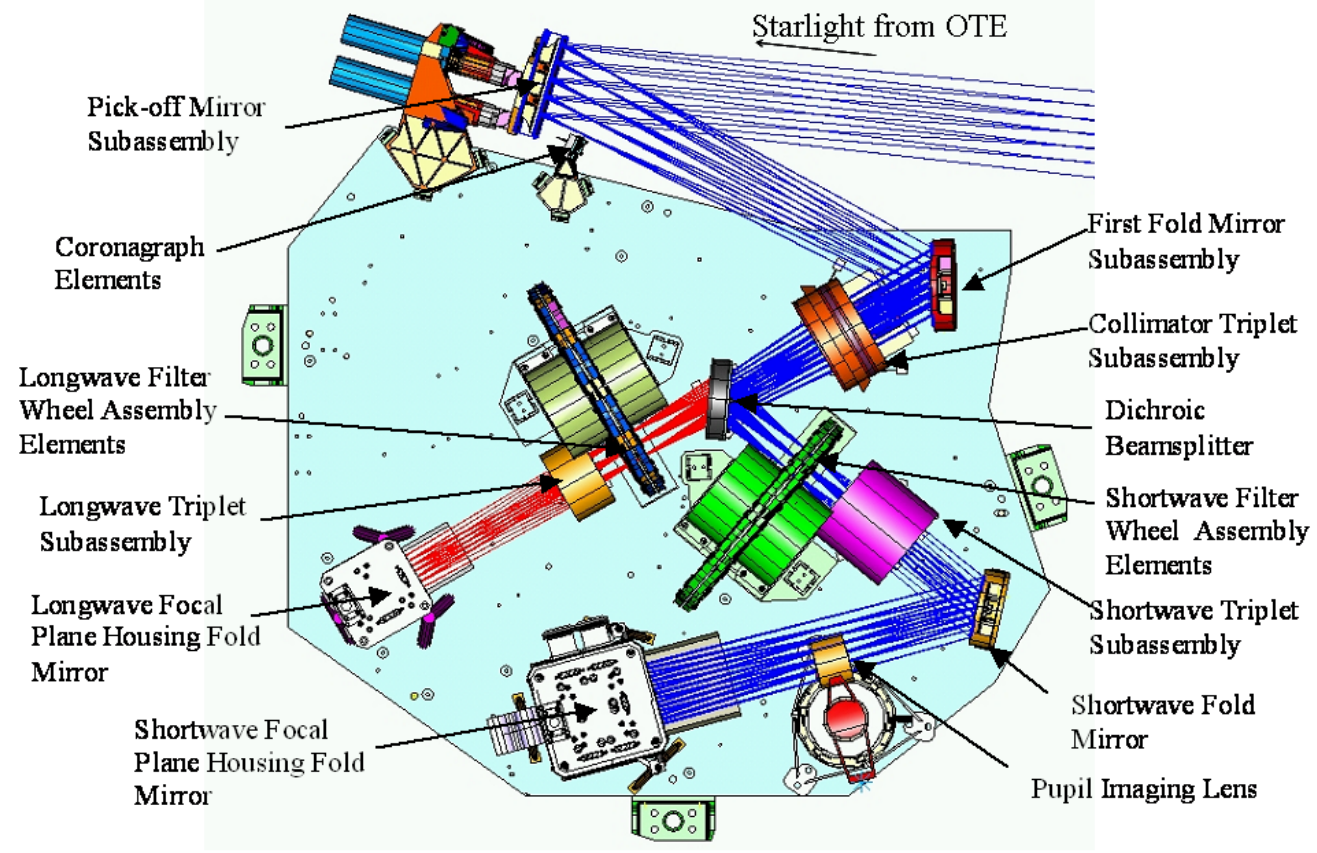

Figure 2: The NIRCam optics train layout

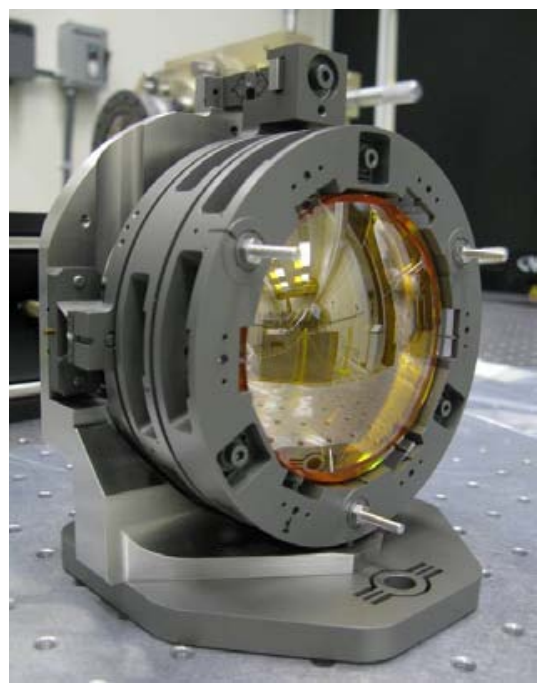

Figure 3: The NIRCam refractive optics design employs advanced high stability cryogenic lens mounts

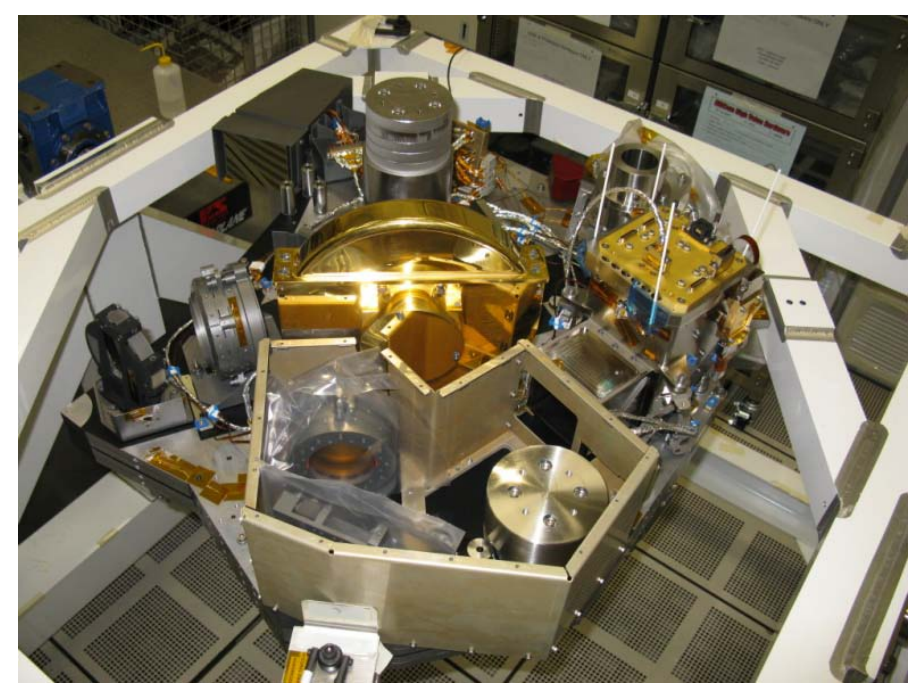

Figure 4: The NIRCam engineering test unit 


\begin{tabular}{|l|l|}
\hline \multicolumn{2}{|l|}{ Table 1: Key NIRCam Features } \\
\hline Wavelength Range & $0.6-5.0 \mu \mathrm{m}$ \\
\hline Optical Design & Refractive with dichroicly channels: 0.6-2.4 $\mu \mathrm{m}$ and $2.4-5.0 \mu \mathrm{m}$ \\
\hline Pixel Scales & 0.0317 '/pixel (short wavelength ), 0.0648”/pixel (long wavelength) \\
\hline Field of View & 4 square arc minutes \\
\hline Spectral Resolution & Multi-filters, $\lambda / \Delta \lambda \sim 4,10,100$ \\
\hline Coronagraphy & Focal plane and pupil masks available for both wavelength ranges \\
\hline Bench & Bonded Be \\
\hline Filter/Pupil Wheels & DC Direct Drive w/ position feedback \\
\hline Pick-off mirrors & Adjustable in focus \\
\hline Detectors & 4Kx4K using $2.5 \mu$ m cutoff HgCdTe, $2 \mathrm{Kx} 2 \mathrm{~K}$ using $5.0 \mu$ m cutoff HgCdTe \\
\hline Redundancy & Fully redundant opto-mechanical design w/ cross-strapped electronics \\
\hline
\end{tabular}

\subsection{The Mid-Infrared Instrument (MIRI)}

Imagery and spectroscopy over the 5-29 micron spectrum is provided by the MIRI instrument ${ }^{9}$. This instrument (Figures 5 and 6) provides broad-band imagery, low (1\%) spectral resolution long slit spectroscopy, and medium $\left(\sim 10^{3}\right)$ spectral resolution integral field spectroscopy (Table 2). The imaging mode includes both occulting and quadrant phase mask coronagraphy. The latter type enables very small inner working angle observations of stellar debris disks and exoplanet systems. When used in combination with the NIRSpec instrument, an optimally sampled integral field spectrum covering the whole 0.6 - 29 micron JWST wavelength range can be obtained at medium spectral resolution.

The MIRI development is a partnership between NASA and the European Space Agency. NASA/JPL is providing a cryo-cooler system to meet the 7K operating temperature requirements of this instrument, a 3 Mpixel Si:As detector system, and overall instrument flight software. The MIRI optical assembly, mechanisms, and instrument-level integration is provided by a European consortium led by the UK Advanced Technology Center and includes the Netherlands, France, Germany, Sweden, Belgium, Spain, Denmark, Ireland, and Switzerland. The MIRI has completed its verification model test program and is currently in flight model integration. Further detail on the MIRI instrument is presented in paper 7731-13 of this conference.

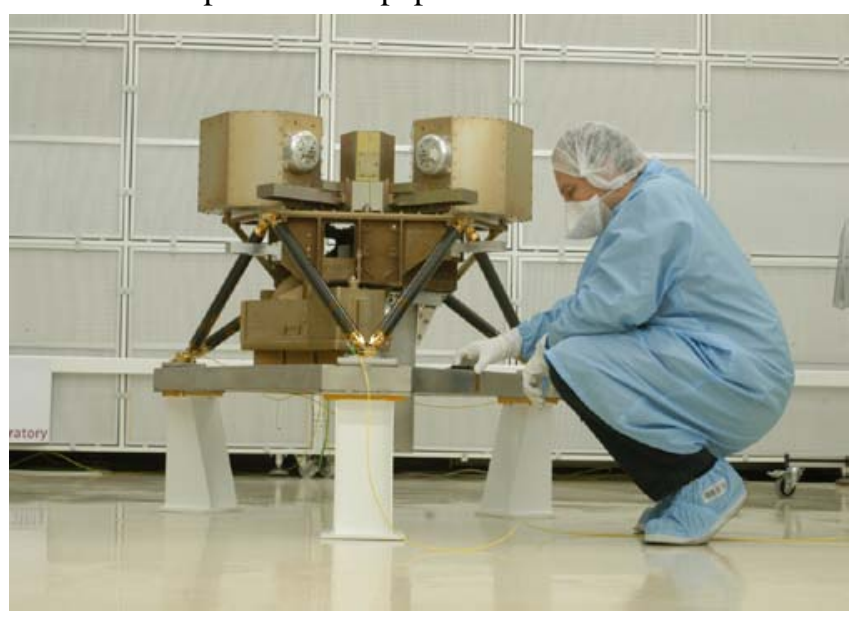

Figure 5: The MIRI structural thermal model

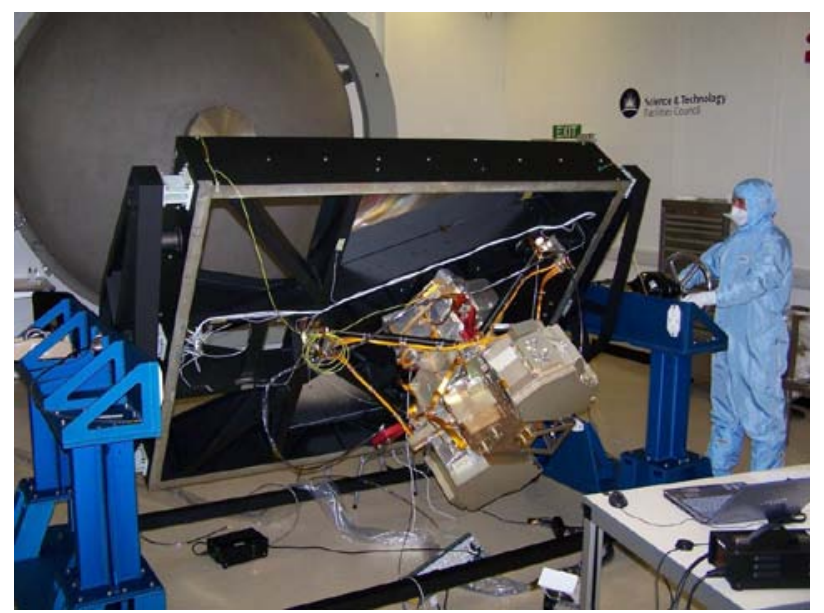

Figure 6: The MIRI verification model

Table 2: MIRI Science Modes 


\begin{tabular}{|l|l|l|l|l|}
\hline Mode & Wavelengths $(\mu \mathrm{m})$ & $\begin{array}{l}\text { Spectral } \\
\text { Resolution }\end{array}$ & Field of View & Comments \\
\hline Imaging & $\begin{array}{l}5.6,7.7,10,11.3,12.8,15,18,21, \& \\
25.5\end{array}$ & $\begin{array}{l}\mathrm{R}=5 \\
\text { typical }\end{array}$ & $1.9 \times 1.4$ arcmin & Discrete filters \\
\hline Coronagraphy & $10.65,11.3,16$, and 24 & $\mathrm{R}=10$ & $25 \times 25$ arcsec & Lyot \& phase mask \\
\hline Spectroscopy & $5-11$ & $\mathrm{R}=100$ & $5 \times 0.2$ arcsec & Long slit \\
\hline Spectroscopy & $5-28.5$ & $\mathrm{R} \sim 2000$ & $\begin{array}{l}3.5 \times 3.5 \text { to 7x7 } \\
\text { arcsec }\end{array}$ & Integral field unit \\
\hline
\end{tabular}

\subsection{The Near-Infrared Spectrograph (NIRSpec)}

NIRSpec is the first multi-object spectrograph to be flown in space. It is designed to obtain near-IR spectra of 100 or more astronomical sources simultaneously at a spectral resolution of $\mathrm{R} \sim 100$ over the 0.6 - 5 micron wavelength range, or at a spectral resolution of $\mathrm{R} \sim 1000$ over 1 - 5 microns. The $\mathrm{R} \sim 100$ mode employs a single prism as its dispersive element and is intended for measuring the redshifts and continuum spectra of faint galaxies. The R 1000 mode utilizes diffraction gratings to cover the 1 - 5 micron spectral region, and is primarily intended for detailed follow-up observations using conventional nebular emission lines as astrophysical diagnostics. Lastly, three R 3000 gratings, also covering 1 - 5 micron, will allow kinematic studies of individual galaxies to be carried out in single object or integral field mode.

The NIRSpec design employs all reflective optics, with most of its optical and structural elements manufactured out of an advanced ceramic material (SiC - 100). The optical chain (Figure 7) has three main components. The foreoptics re-image and magnify the focal plane image of the JWST telescope onto the slit selection mechanism. The collimator converts the light emerging from each slit into a parallel beam and projects it onto the grating wheel which carries six (flat) reflective gratings, a (dual pass reflective) prism and a (flat) mirror for target acquisition. The camera finally focuses the dispersed collimated light coming off the grating onto the detector array. A filter wheel located at an internal pupil of the fore-optics carries order separation filters for the diffraction gratings and also serves as the instrument shutter.

The NIRSpec multi-object aperture control (Figure 7) is a programmable Micro-Shutter Array (MSA) provided by the Goddard Space Flight Center (GSFC). The MSA is made up of four sub-arrays, each comprising 365 x 171 individually programmable shutters. The open area of each shutter is 200 mas wide and 460 mas long. The active area of the whole MSA spans a field of view measuring 3.4 x 3.6 arc-minutes on the sky. In addition to the programmable micro shutters, the MSA also carries several fixed slits that can be used for high contrast observations of single objects at any of the three spectral resolutions, and an integral field unit for use with three $\mathrm{R} \sim 3000$ gratings.

The NIRSpec detector system, also provided by GSFC, consists of two close-butted 2k x 2k Rockwell HgCdTe detector arrays. In order to optimize the detector noise limited sensitivity of NIRSpec, the detector samples the spectra at a relatively coarse 100 mas per pixel.

NIRSpec is being built by the European Space Technology Center with EADS Astrium. See paper 7731-12 of this conference for further details. 

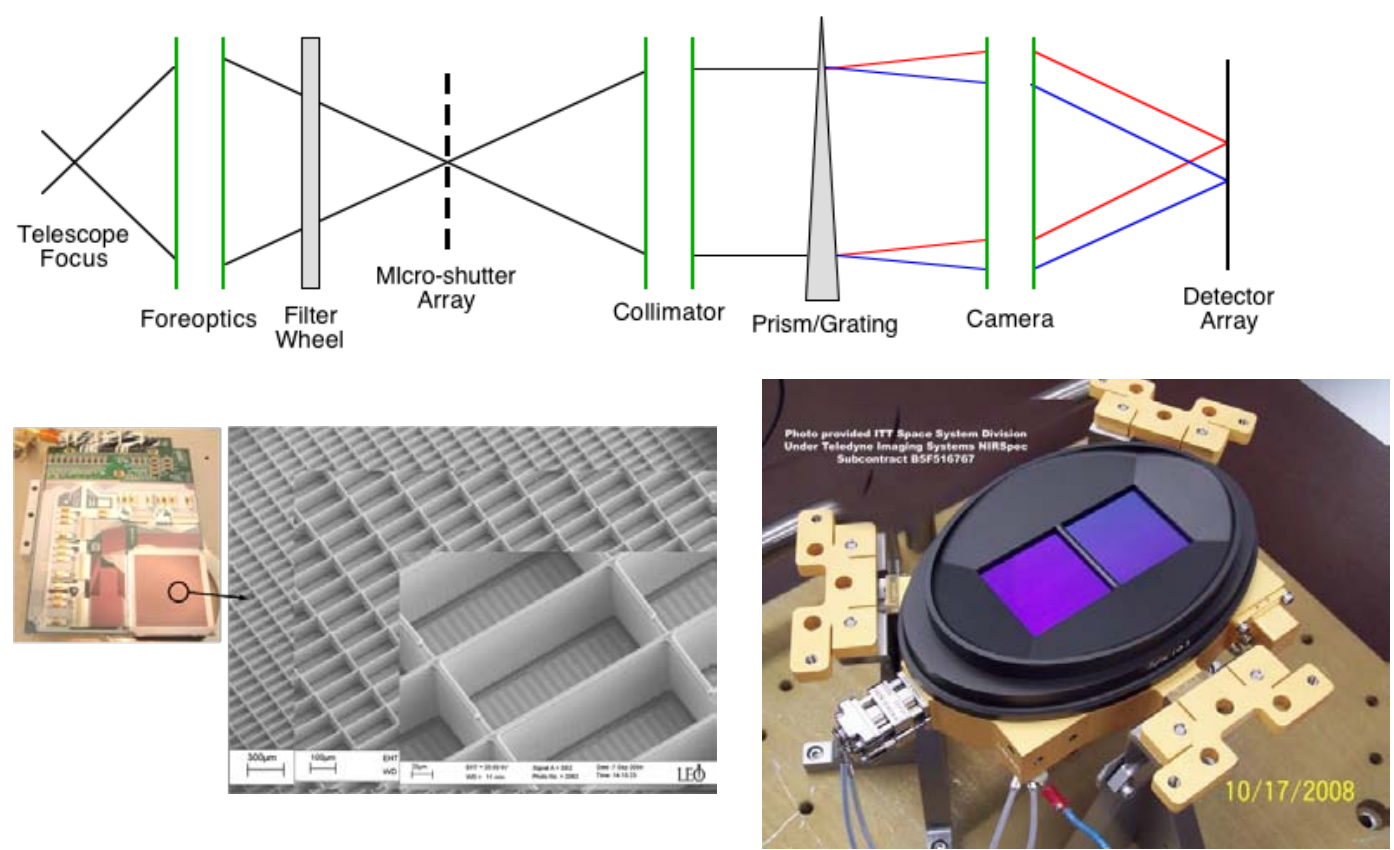

Figure 7: NIRSpec optics schematic (top) showing the position of the micro-shutter array (lower left) and detector arrays (lower right) in the optics train.

\subsection{The Fine Guidance Sensor (FGS) and Tunable Filter Instrument (TFI)}

In order provide sensing for the observatory's 7 mas line-of-sight stability control, an image-based fine guidance sensor is located in the ISIM with the science instruments. The optical assembly of the FGS instrument (Figure 8) consists of two fully redundant modules. These modules image two adjacent fields of 2.3' on a side, with 68 mas pixels. This scale will enable the required guiding precision of 4.9 mas at $16 \mathrm{~Hz}$, with a faint limit that gives $95 \%$ guide star probability in the emptiest part of the sky. The two redundant detector systems are fed by a single set of relay optics, and operate over a wavelength range of 0.6 to 5.0 microns.

The TFI shares an optical bench with the FGS. It consists of a low order Fabry-Perot tunable filter that images a similar field of view (2.2') with a 65 mas pixel scale and operates at a resolving power of approximately 100 over the 1.5 to $5.0 \mu \mathrm{m}$ spectral range with a non-functional region between 2.6 and $3.1 \mu \mathrm{m}$. The TFI optics image a single order of interference onto the focal plane array such that the scanning etalon produces a spatially resolved spectra of all objects in the field of view. Coronagraph spots at the edge of the field provide central light suppression of $10^{-4}$ at 1 arc-sec radius. Further detail on the FGS and TFI instruments is given in paper 771-14 of these proceedings. 

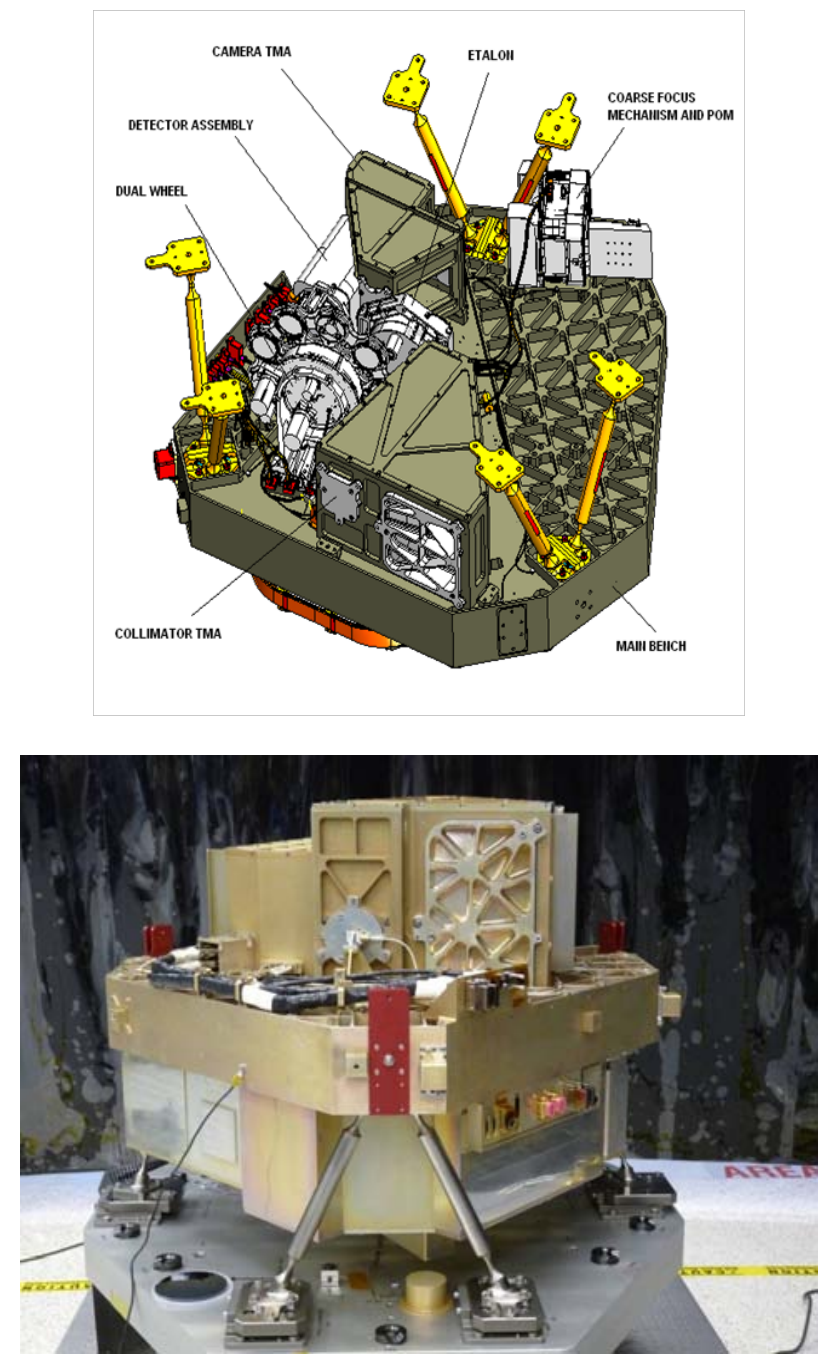

Figure 8: The layout of the TFI on one side of the FGS bench (top) and the ETU of the combined Guider and TFI (bottom)

\section{SCIENCE SUPPORT SYSTEMS}

\subsection{Optical Metering Structure System}

The ISIM Optical Metering Structure (Structure) is a state-of-the-art bonded composite structure, and is the first large (2.5m length) cryogenic optical metering structure to be built and tested for JWST (Figure 9). The Structure houses the Science Instruments (SI), provides structural support for ground testing and launch, and maintains the correct position of the science instruments with respect to each other and the Optical Telescope Element (OTE) to a precision of approximately 80 microns upon cryogenic cycling and launch loading. It is a three-dimensional frame of $75 \mathrm{~mm}$ cross-section square tubes made of carbonfiber/cyanate-ester composite material, bonded together with gussets made of composite and clips made of Invar 36. Large node fittings made of Invar 36 are used in a few select locations, most notably at the interfaces to the OTE and to Ground Support Equipment (GSE). The SIs are supported by saddle fittings made of Invar 36 bonded to the tubes, and attached via Titanium Interface Plates that are individualy customized to provide precise alignment of the Instruments with respect to each other and to the Telescope. Composite material was selected for high specific stiffness and low Coefficient of Thermal 
Expansion (CTE). Invar 36 was selected for low CTE, but at the cost of low strength and high density. Titanium was selected for high specific strength. The tube topology was driven by OTE and SI accommodation; stable attachment to the OTE near the primary mirror, stable attachment of the SIs near the light coming from OTE, and the limits of physical space available within the overall JWST architecture.

The key design challenges were to support the SIs in precise alignment, maintain alignment stability through 270 degrees of temperature with cryogenic cycling, survive the large loads applied during rocket launch, survive exposure to extreme cryogenic temperatures (27K), and distort during cool-down from room temperature to cryogenic temperatures less than $0.5 \mathrm{~mm}$. In addition, critical JWST optical performance predictions are made using mathematical models, including a math model of the ISIM Structure. Accurate correlation of the Structure thermal distortion math model to measured thermal distortion test results was required and has been achieved on the flight structure.

To address these challenges, the project executed a comprehensive 'building block' development program to measure cryogenic cool-down distortion, room temperature strength, and cryogenic temperature strength of the constituent materials and structural elements at the following successively higher levels of assembly: 1) composite lamina/laminates, adhesives, and metals, 2) composite tubes, 3) bonded tube/gusset/clip joints and bonded tube/fitting joints, 4) a representative bonded frame, and finally 5) the flight Structure. All of the non-flight development work needed to validate the flight design and correlate the Structure thermal distortion math model concluded in 2008, the Flight Structure build was completed in 2009, and cryo-distortion testing of the Flight Structure at GSFC completed in May 2010 (Figure 9). Photogrammetric measurements with $>0.025 \mathrm{~mm}$ accuracy were made at room temperature and cryogenic operational temperature, demonstrating that Structure cool-down distortions are well within the required $0.5 \mathrm{~mm}$ limit. This is a major risk reduction milestone, and a major success for the JWST program.
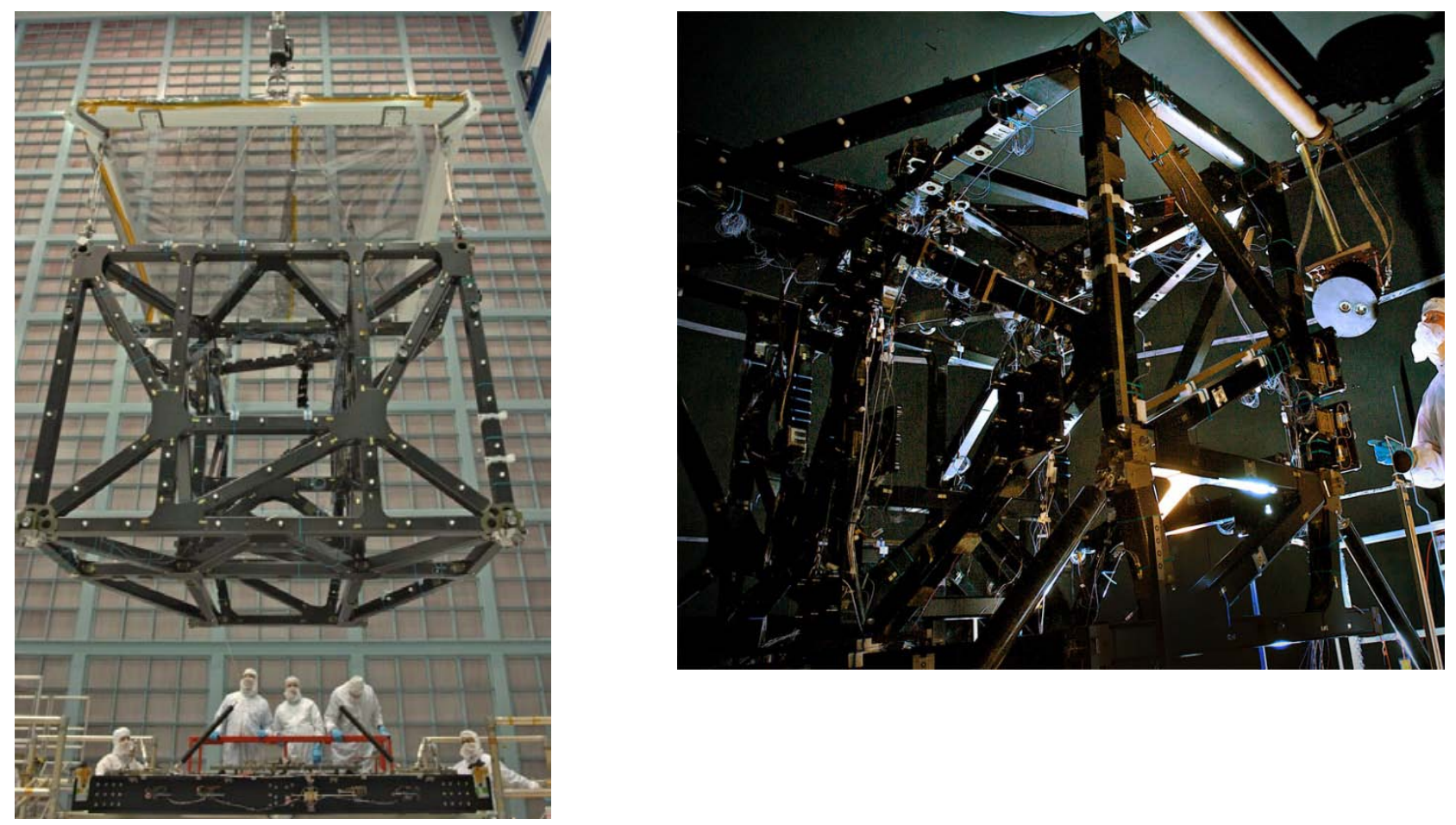

Figure 9: The ISIM flight structure in the SSDIF clean room (left); and in the space environment simulation chamber for cryogenic photogrammetric testing (right). 


\subsection{ISIM Electronics Compartment (IEC)}

The ISIM Electronics Compartment (IEC) is one of the primary engineering challenges of the observatory. It accommodates warm (300K) instrument electronics that must reside close to the cryogenic (40K) science instruments due to cable length restrictions. As a result, it must be located on the cryogenic side of the sunshield (Figure 10). The eleven electronics boxes in the IEC dissipate a total of 230W which must be rejected to space such that the heat leak (both radiative and conducted by structural interfaces and electrical harnesses) to the surrounding cryogenic system is controlled to approximately $350 \mathrm{~mW}$ representing roughly $1 / 3$ of the total ISIM cryogenic heat load to the Observatory.

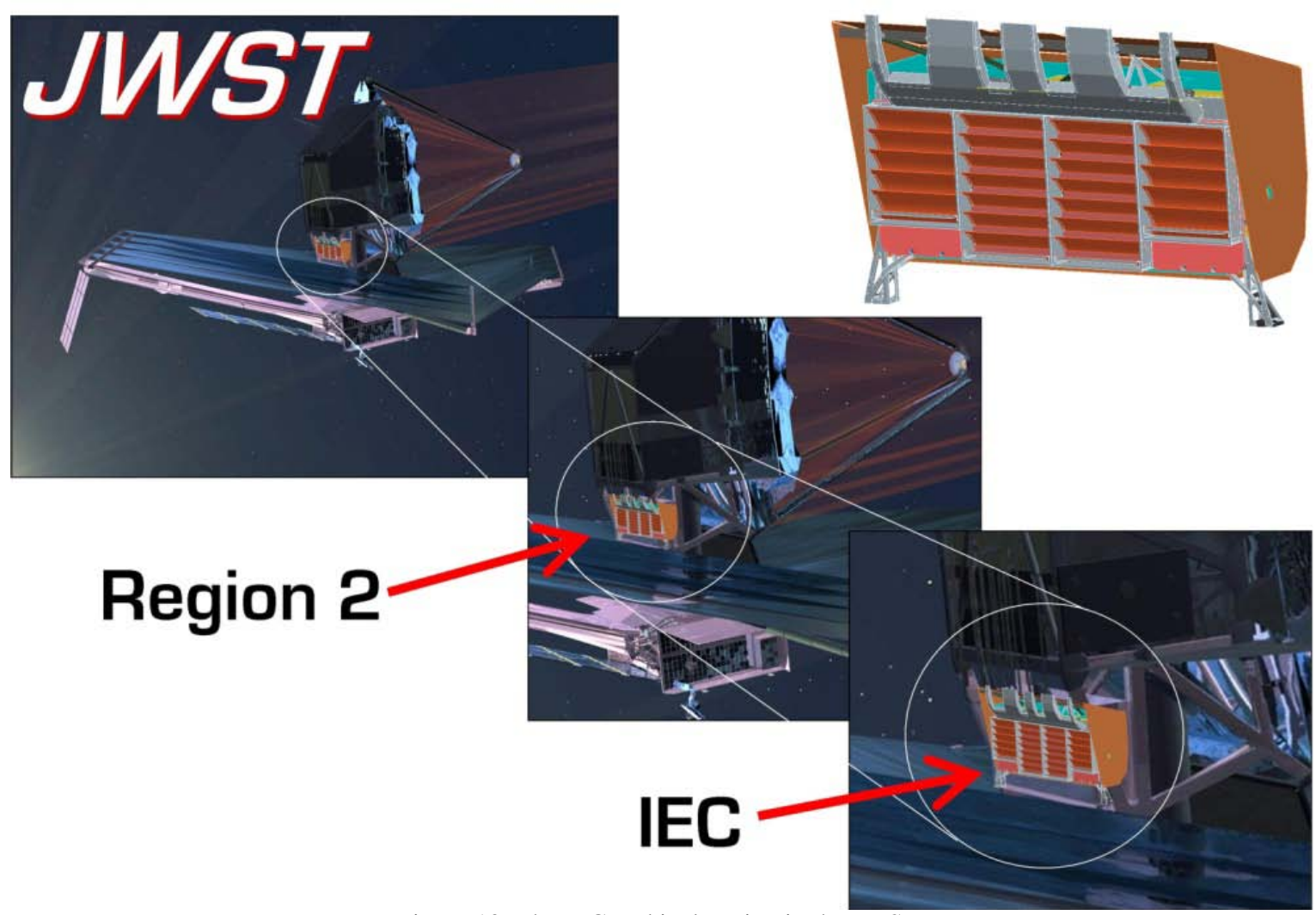

Figure 10: The IEC and its location in the JWST

Control of the IEC power dissipation is critical to the operation of the JWST. Precise control of heat leaks is essential to achieving thermal balance of the telescope and instruments as well as avoidance of infrared stray light. The IEC power is rejected to space via a flat panel radiator that is located behind an array of composite cylindrical mirrors (baffles) to create a beam pattern that avoids impingement on adjacent cryogenic surfaces (Figure 11). 


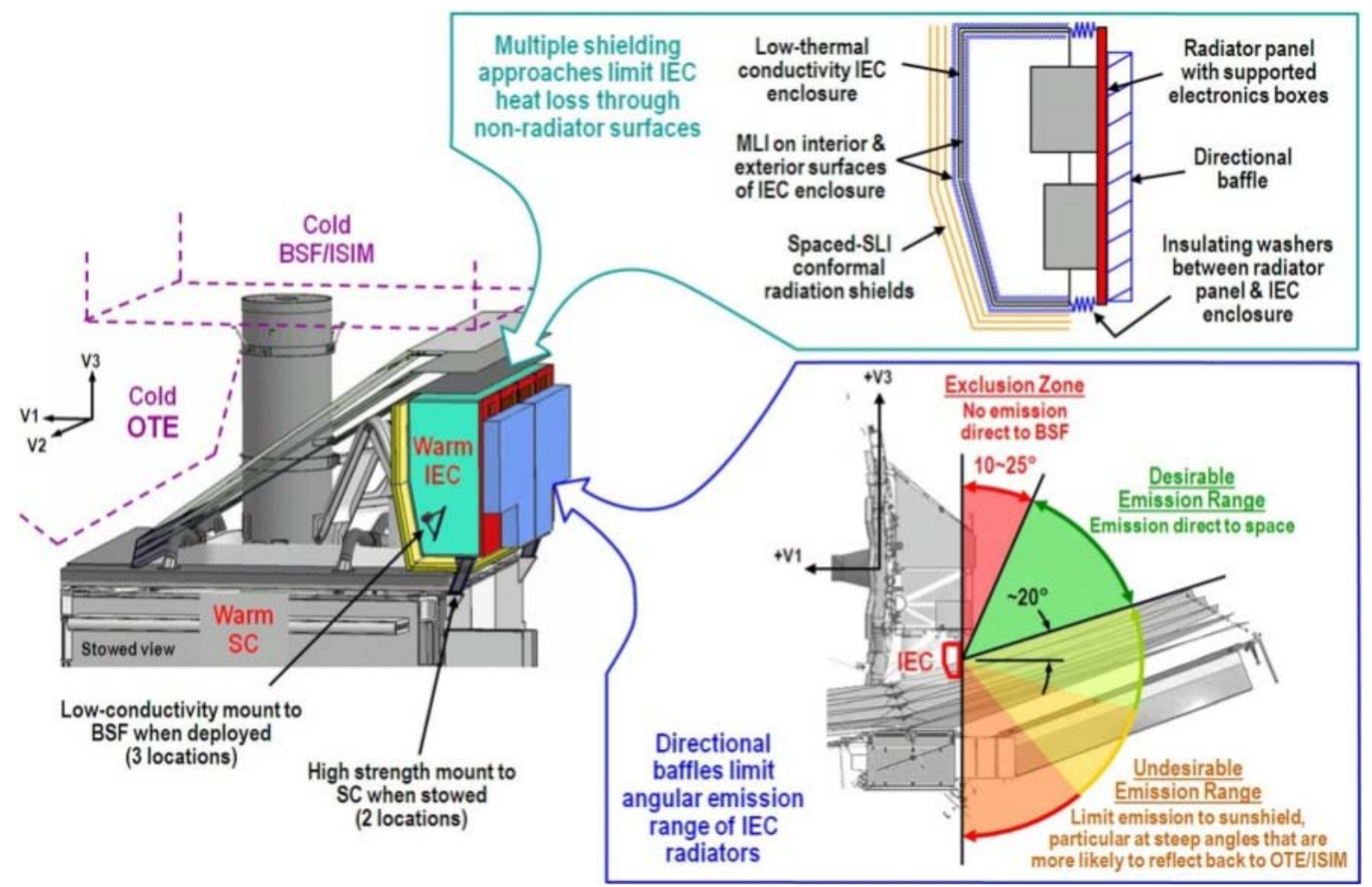

Figure 11: The IEC radiators must emit with a 20 deg beam pattern

The IEC protective enclosure utilizes composite technology (Figure 12) the "Shell" is fabricated with from composite construction that is robust and can properly house the electronics boxes and yet strategically emit the heat so it does not interfere with the science equipment. The shell is primarily made of fiberglass and Nomex ${ }^{\circledR}$ honeycomb core. The baffles incorporate a unique gold vapor deposition process over its fiberglass structure to give it a low emissivity surface. The radiator panels are constructed of expanded aluminum honeycomb core sandwiched by two thin face-sheets. The majority of the components for the IEC are fabricated with integration and test underway (Figure 13).
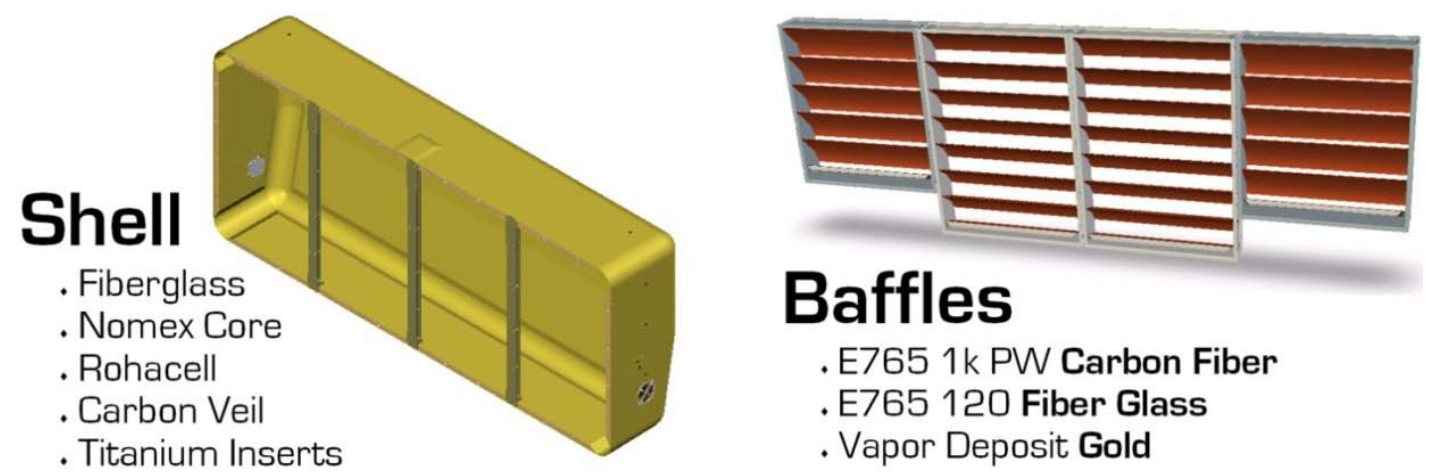

.E765 1k PW Carbon Fiber

. E765 120 Fiber Glass

- Vapor Deposit Gold

Figure 12: Composite Materials are used to fabricate the IEC 

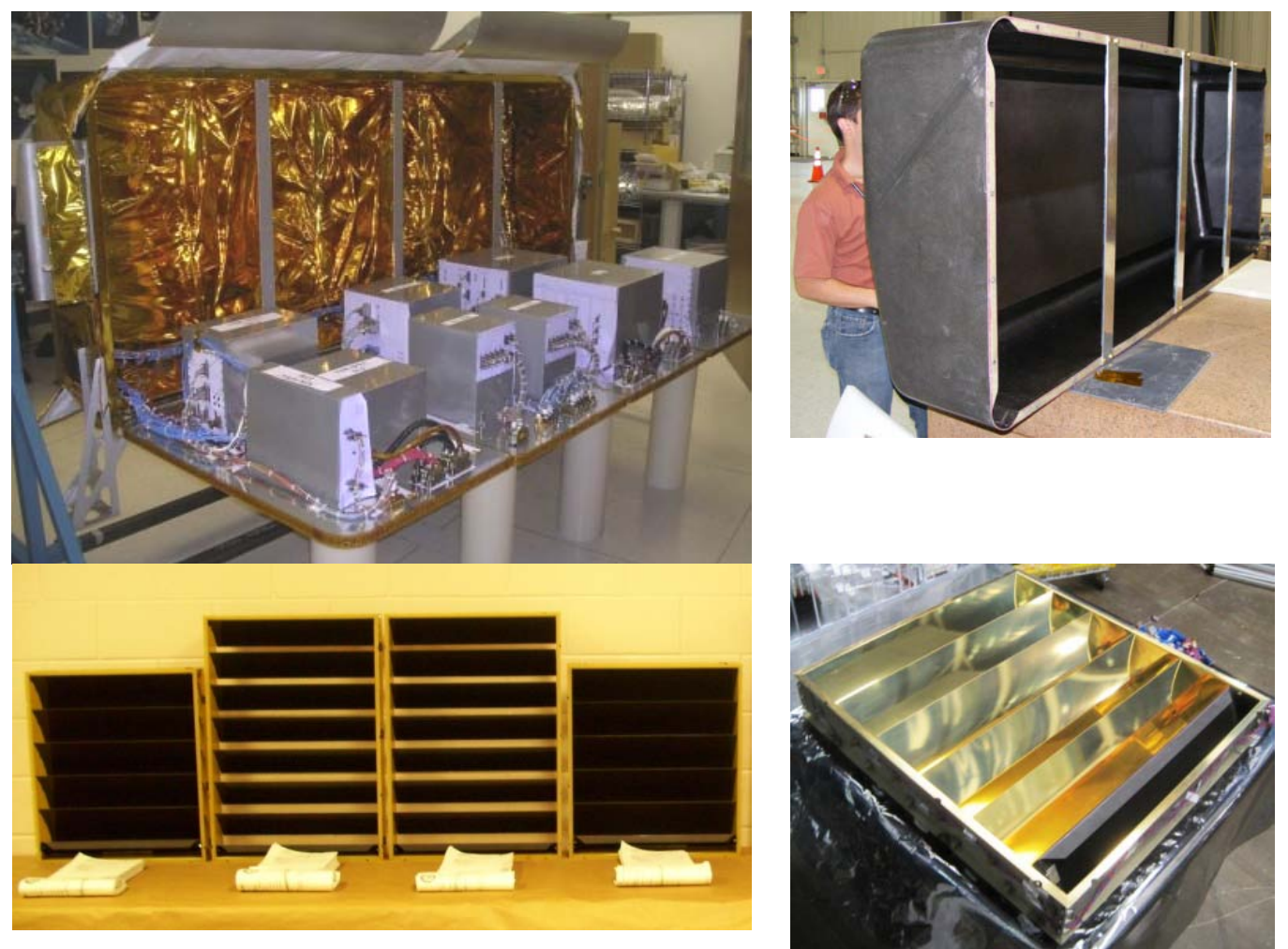

Figure 13: Full scale mock-up of the IEC showing electronics boxes integrated to the interior surface of the IEC flat panel radiator (top left), the flight IEC enclosure (top right), and the flight baffle mirror assemblies (bottom).

\subsection{Harness Radiator System}

The harness radiator component of the ISIM system was a late addition to the architecture (Figures 14 and 15). It was added after the IEC was implemented and is intended to capture and radiate to space the parasitic heat flowing in electrical harnesses attached to the electronics within the IEC. These electronics are standard designs and operate in the -20C to 40C range. This warm set of electronics is in close proximity $(\sim 2 \mathrm{~m})$ to the cryogenic instruments. As a consequence, it is necessary to intercept the heat from these short thermal transition harnesses and radiate it to space with a dedicated radiator system.

A detailed set of requirements for the Harness Radiator (HR) have been developed and are being implemented. Among these are its overall mass, the launch and testing environments, and mechanical interface loads. Three of the principle performance requirements will be described here.

The most critical performance requirement for the HR is the allowable heat load to the instruments and telescope due to the harnesses. This heat load is used to establish the heat intercept needs of the Harness Radiator. The total allocated heat load to the ISIM cryogenic section is $460 \mathrm{~mW}$. Of this, an allocation of $150 \mathrm{~mW}$ has been given to the electrical harness that are managed by the HR. The HR is therefore required to reduce the heat load below this $150 \mathrm{~mW}$ value. The harness radiator must also limit the total heat load to the Optical Telescope Element, and the Backplane Support Fixture (BSF) to less than $30 \mathrm{~mW}$. At present, the HR design is predicted to reduce the heat load to the instruments to less than $95 \mathrm{~mW}$.

A second key performance requirement is the need to accommodate the relative motion of the ISIM, the telescope (BSF), and the IEC (Figure 15). Each of these components are mounted on kinematic (or iso-static) mounts. So motion between these components will be seen both during launch, and during the system cool-down to reach on orbit operating temperatures. The requirement for this displacement is to accommodate $18 \mathrm{~mm}$ of relative motion between the HR and the BSF. 
The third key performance requirement is to shield the electrical harnesses from micro-meteor (MM) damage. To achieve the best thermal performance of the HR and the harnesses, the HR design routes the harnesses external to the set of enclosures in which the instruments are mounted. This routing provides the best view to space for the harnesses but also exposes the harnesses to potential damage from micro-meteors. An extensive study of the behavior, performance, and potential damage mechanisms, of exposed harnesses was conducted by the NASA Engineering Safety Council (NESC). A set of study guidelines and questions regarding micro-meteor damage and performance were developed by the ISIM engineering team and provided to the NESC for evaluation. The NESC team reviewed past mission harness performance, conducted analysis of the MM environment, determined the damage potential of the harnesses based on these studies, and conducted hypervelocity MM tests of representative electrical harnesses. The recommendation of the NESC was that harnesses routed external to the ISIM enclosure should be shielded by 1 to $2 \mathrm{~mm}$ of titanium in order to protect the harnesses and meet the overall probability of success rates imposed on the harnesses. This recommendation has been implemented.

The HR accommodates two basic types of electrical harnesses. These are the instrument detector interface harnesses, and the instruments engineering (lamps, mechanisms, heaters, temperature monitoring) interface harnesses. The detector interface harnesses are flat laminated cables composed of fine gauge wires (36 AWG and smaller). The engineering interface harnesses are also fine gauge wires (26 AWG and smaller) but are of a more standard wire harness construction using kapton and Teflon insulted harness bundles.

To improve the ability to draw heat from the harnesses and radiate it to space, the HR has implemented four distinct radiator panels each mounted to a common backbone support structure. As the harnesses cross over each of these panels, a harness clamp has been implemented which compresses the harnesses to a controlled compression value. Testing has been done to show the harnesses survive and properly function while compressed at clamp pressures up to 200 psi. Because there is differential contraction of the harnesses as well as the different panels of the Harness Radiator, strain relief loops/hoops are designed into the harness sections between the harness clamps.

The Harness Radiator has been designed to allow harnesses for the four science instruments to be separately integrated in any order. This has been done to improve overall system integration and testing workarounds by allowing one instrument to be removed, with its harness, while another instrument can continue testing.

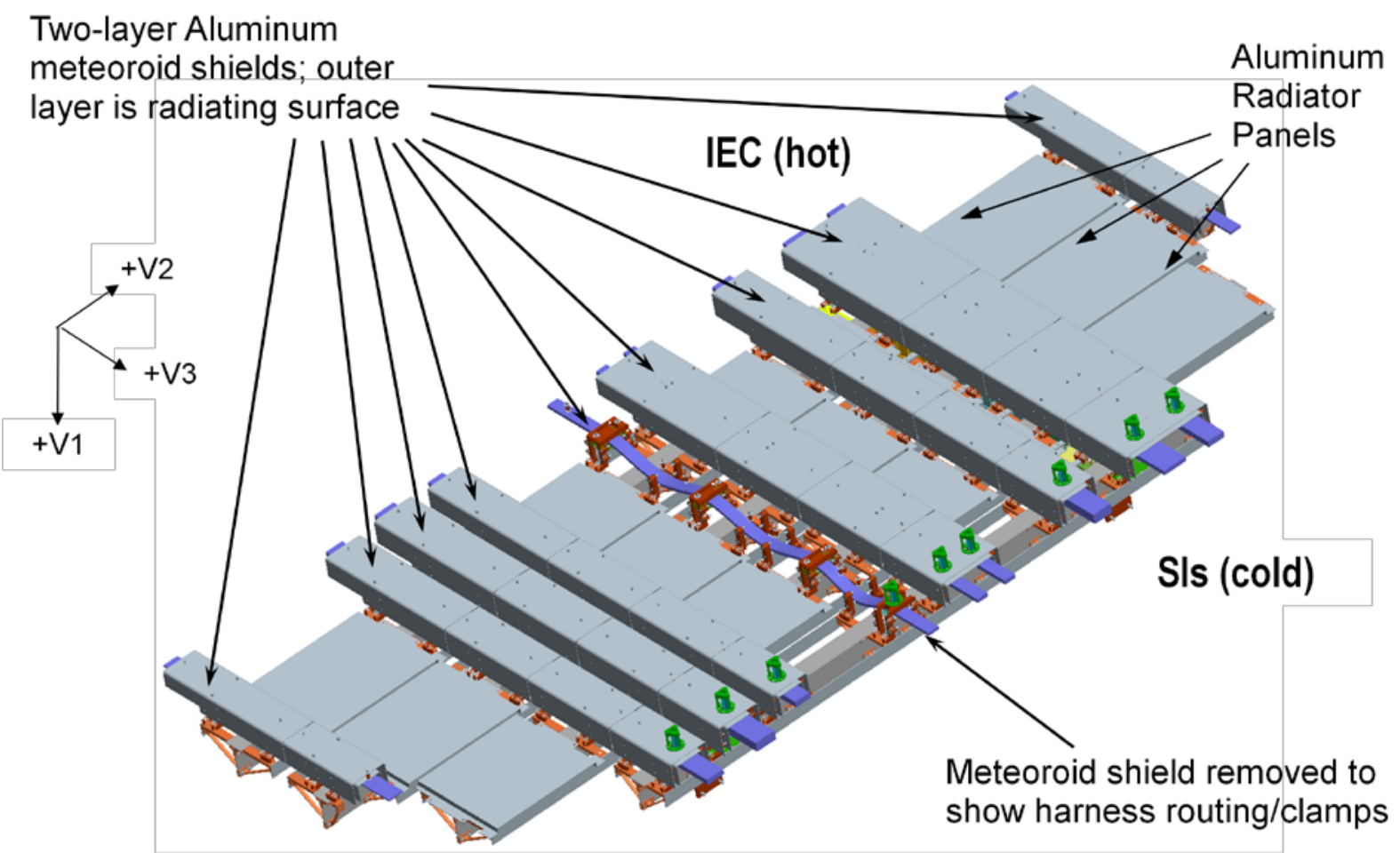

Figure 14: The harness radiator assembly 


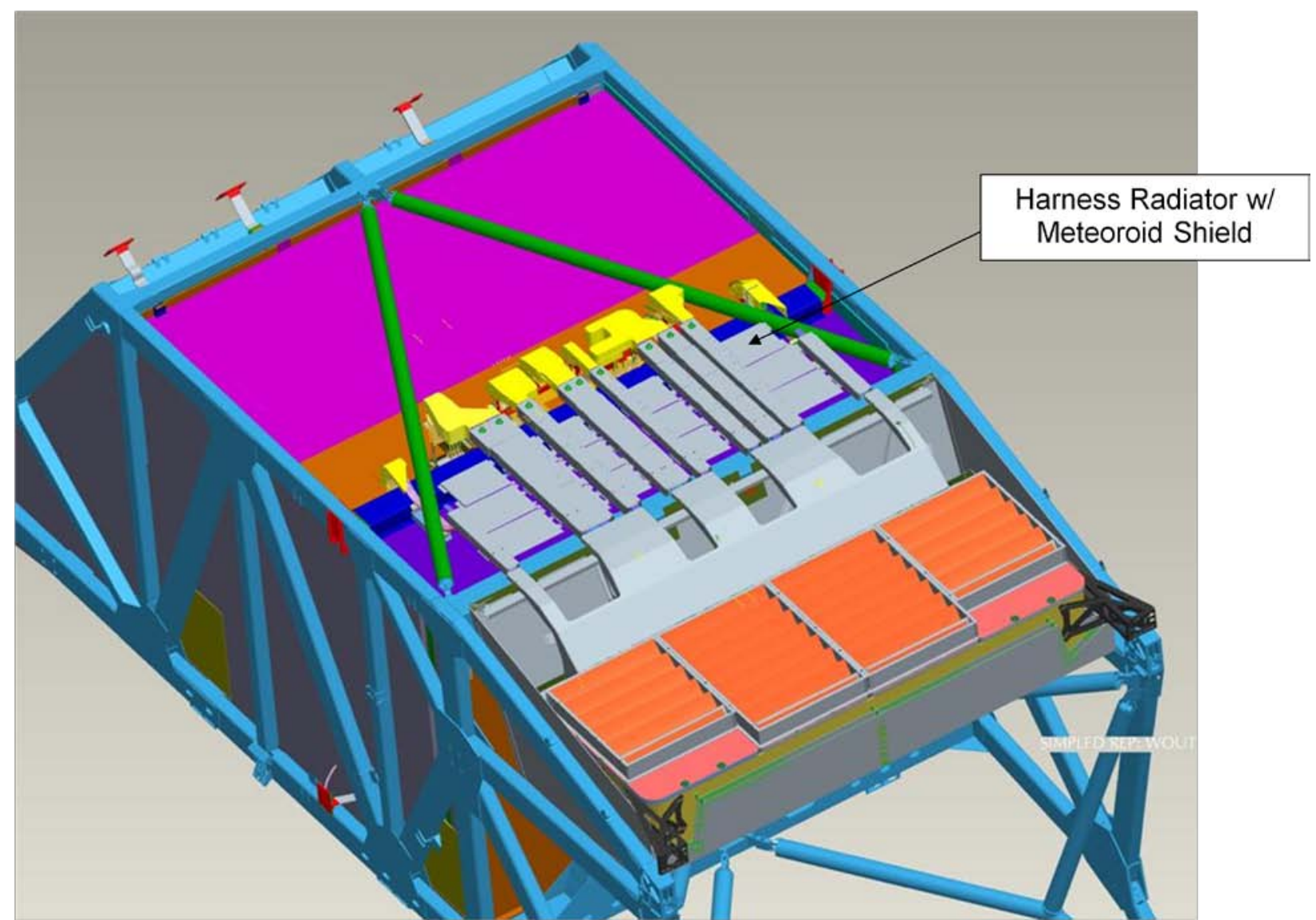

Figure 15: The harness radiator system shown along with the ISIM cryogenic assembly, the IEC , and telescope BSF structure.

\subsection{Command \& Data Handling and Remote Services Subsystems}

Figure 16 shows the ISIM Command and Data Handling (IC\&DH) and Remote Services Unit (IRSU) in context of the overall ISIM electrical system. The IC\&DH/IRSU hardware and software together provide four major functions: [1] Coordinate collection of science image data from the NIRCam, NIRSpec MIRI, and FGS instruments in support of science objectives; [2] Perform multi-accum \& lossless compression algorithms on science image data to achieve data volume reduction needs for on-board storage and subsequent transmission to the ground; [3] Communicate with the Spacecraft Command and Telemetry Processor (CTP) to transfer data to the Solid State Recorder (SSR) and prepare for subsequent science operations; and [4] Provide electrical interfaces to the Thermal Control Sub-system.

The IC\&DH hardware consisting of 7 separate assemblies is shown in Figure 17 along with the general science image data collection, processing and storage flow. The IRSU consists of 3 assemblies and is shown in Figures 18. 


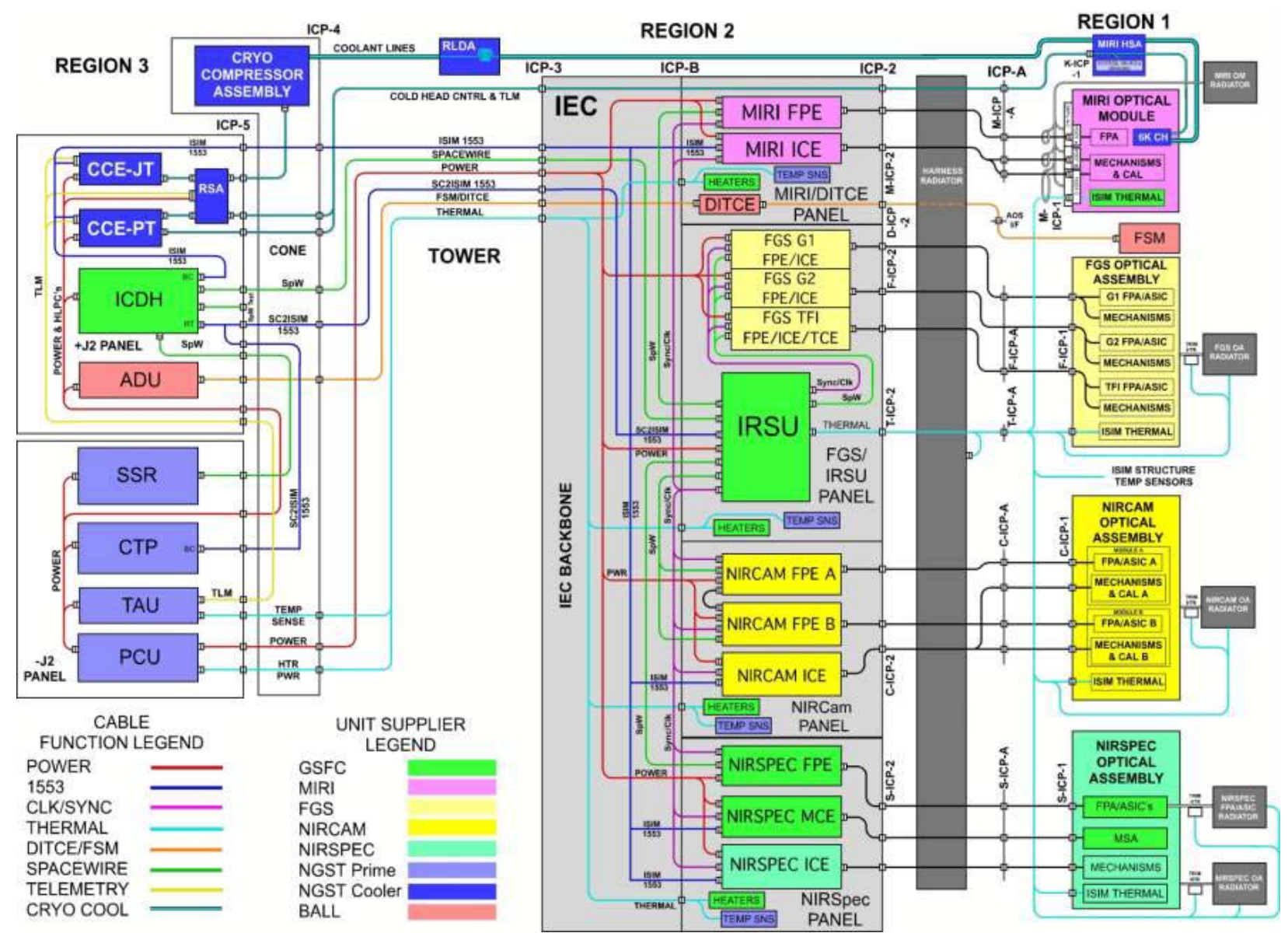

Figure 16: ISIM Electrical Interface Diagram

The IC\&DH/IRSU communicate with the Instruments over Spacewire Point-to-Point interfaces operating at link rates between 9.4 Mbps to 60 Mbps. The IRSU contains a Multi - SpaceWire Concentrator Card (MSC) that interfaces via SpaceWire to the instruments and concentrates and routes the packets to the IC\&DH sub-system. The internal SpaceWire communications within the IC\&DH occur at a link rate of 80 Mbps.

The IC\&DH provides the centralized ISIM Command and Data Handling functions for the NIRCam, NIRSpec, MIRI, and FGS instruments and unifies the Image collection with a common Spacewire point to point topology. The JWST IC\&DH is unique in that it centralizes the C\&DH functions for NIRCam, NIRSpec, MIRI, and FGS away from the detector electronics region in order to manage stringent thermal requirements while maintaining the ability to receive image data at peak detector readout rates. Multiple SpaceWire 4-port Routers within the IC\&DH and IRSU provide high speed extraction of image data from each of the instruments and data routing to each of the Focal Plane Array Processors (FPAP). The FPAPs are unique in that they are dynamically configurable and assignable to any one of the 18 total NIRCam, NIRSpec, MIRI, and FGS instrument Sensor Chip Assemblies.

The IRSU also provides several electrical functions for the Thermal Control Sub-system. IRSU provides power to the heaters used to perform contamination control and to operate the Instrument Trim heaters. Also, IRSU interfaces to temperature sensors within ISIM and will perform readout of those sensors, relaying that data to the S/C for downlink. The IC\&DH/IRSU interfaces to each of the Instruments support unique and independent science operations while providing a standard protocol across the interface ultimately resulting in integration and test risk reduction during ISIM Integration and Test. 


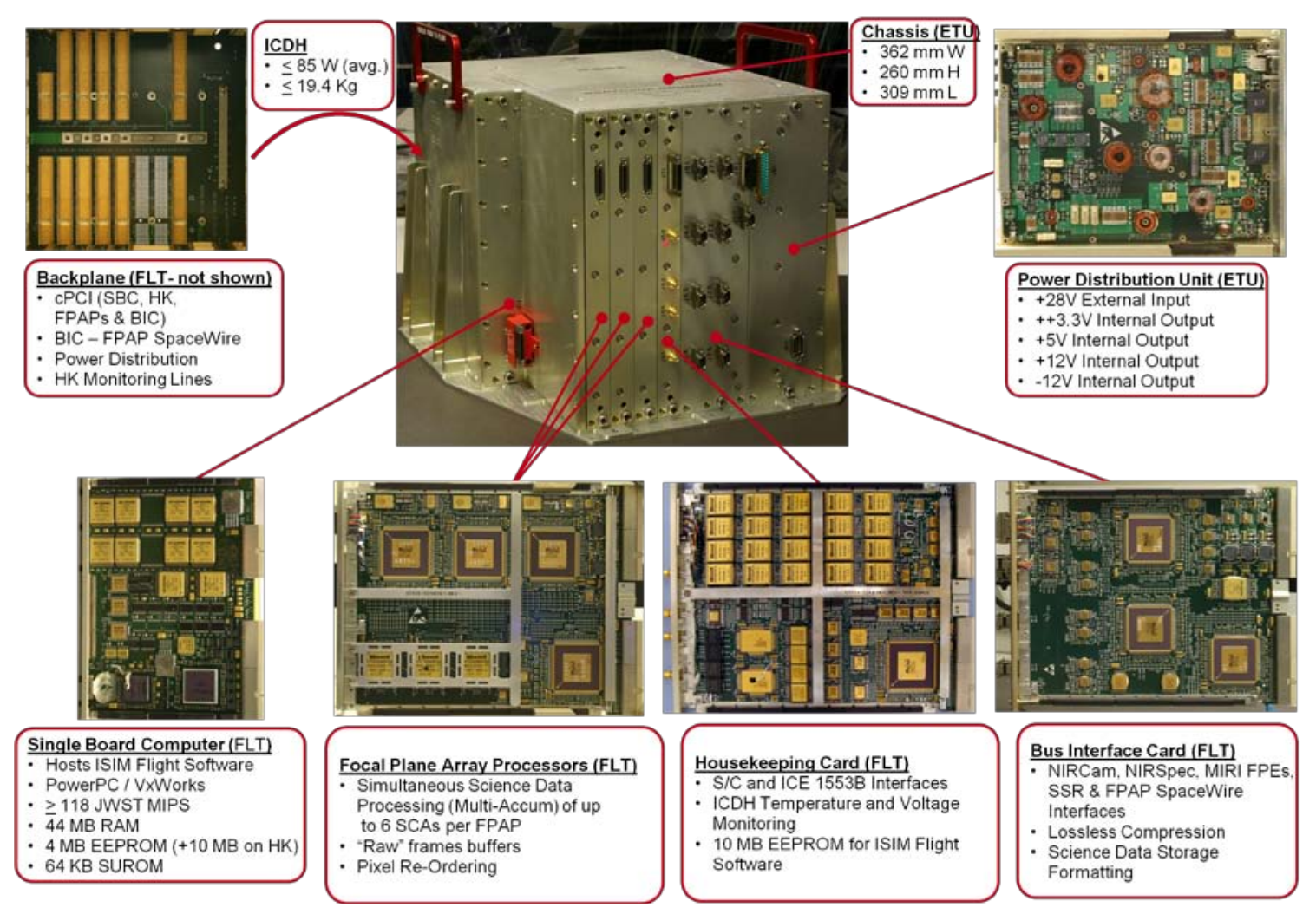

Figure 17: The flight IC\&DH Assembly

Finally, the IC\&DH/IRSU has been architected to meet high data throughput and processing requirements while minimizing overall lifecycle costs and risks. Both ICDH and IRSU Flight Units have been fabricated and are undergoing final environmental tests prior to delivery.

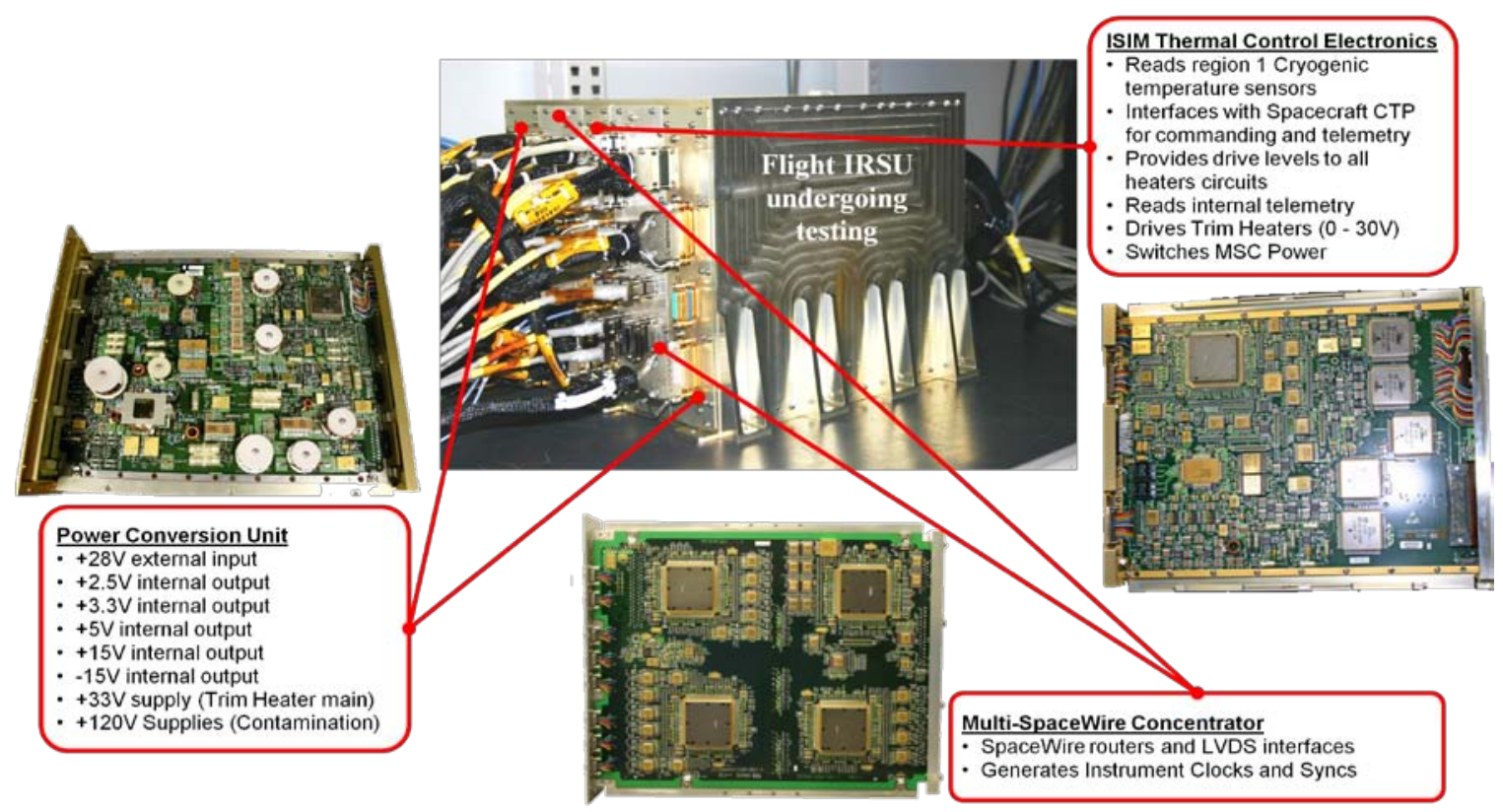

Figure 18: The flight IRSU assembly 


\subsection{Cryogenic Thermal Control System}

The cryogenic portion of the ISIM lies on the anti-sun side of the large sunshields, permanently shaded from the sun, the warm spacecraft bus and solar panels. When operating, the detectors and optics of the three Near-infrared Instruments must be cooled to the range of approximately 36 to $40 \mathrm{~K}$ and the Mid-Infrared Instrument (MIRI) must be cooled to approximately $6 \mathrm{~K}$. In order to support long integration observation of faint objects, the thermal environment of the instruments must also be kept stable to within $0.1 \mathrm{~K}$ and $0.25 \mathrm{~K}$ over 10,000 seconds, and 24 hours respectively. Additionally, thermal isolation of the science instruments from each other enhances their stability and maximizes science data return. To accomplish these key requirements, the entire instrument suite is passively controlled to this temperature range via direct instrument coupling to dedicated radiators. Individual thermally isolated radiators are provided to cool the NIRCam, FGS, and NIRSpec instruments, the MIRI electrical harnesses and the bases of the MIRI support struts. The instruments are connected to their dedicated radiators through a complex series of segmented high purity aluminum heat straps designed to minimize the thermal gradient between instrument and radiator. These flexible aluminum straps are supported in composite channels, which are mounted to the ISIM structure and the surrounding BSF through composite flexures. Figure 19 shows a photo of three proto-flight strap segments with support flexures, prior to vibration testing at the vendor.

A two stage mechanical cooler maintains the MIRI at its operating temperature of approximately $6 \mathrm{~K}$, as this temperature is too cold to be achieved using only passive techniques. Tests performed at the MIR instrument provider, combined with thermal analyses performed at the GSFC, recently indicated that the cooler's 6K cold stage would have had insufficient heat lift margin when the instrument is immersed in ISIM's 40K thermal environment. As a result, the ISIM is also providing a thermal radiative shield around the MIR instrument, cooled through direct contact with the cooler's second stage heat exchanger operating at approximately $20 \mathrm{~K}$. The resulting trade of heat loads between the cooler's second and third stages raises the performance margin on the 6K stage to acceptable levels. Measures taken to assure the ISIM compartment reaches its designated operating temperatures include assignment of milliwatt-level heat load allocations to each of the instrument optical assemblies, and establishment of interface temperature requirements with the Observatory. Additionally, extensive efforts have been devoted to the design and detailed analysis of the Harness Radiator (see Section 3.3), a multi-stage radiator designed to reject most parasitic heat from the instrument harnesses to space as they enter the cold ISIM from the ambient temperature IEC (see Section 3.2).

The carbon-based composite ISIM structure is a potential source of entrapped water. A contamination avoidance cool-down procedure has been devised to avoid contamination of the instrument detectors and sensitive optics during the instrument cool-down following launch: contamination control heaters on the instruments will be activated to keep them warmer than $165 \mathrm{~K}$ until the entire ISIM structure cools to below $140 \mathrm{~K}$, entrapping the moisture within it or on non-sensitive surfaces. Once the instruments reach operational temperatures, it would take

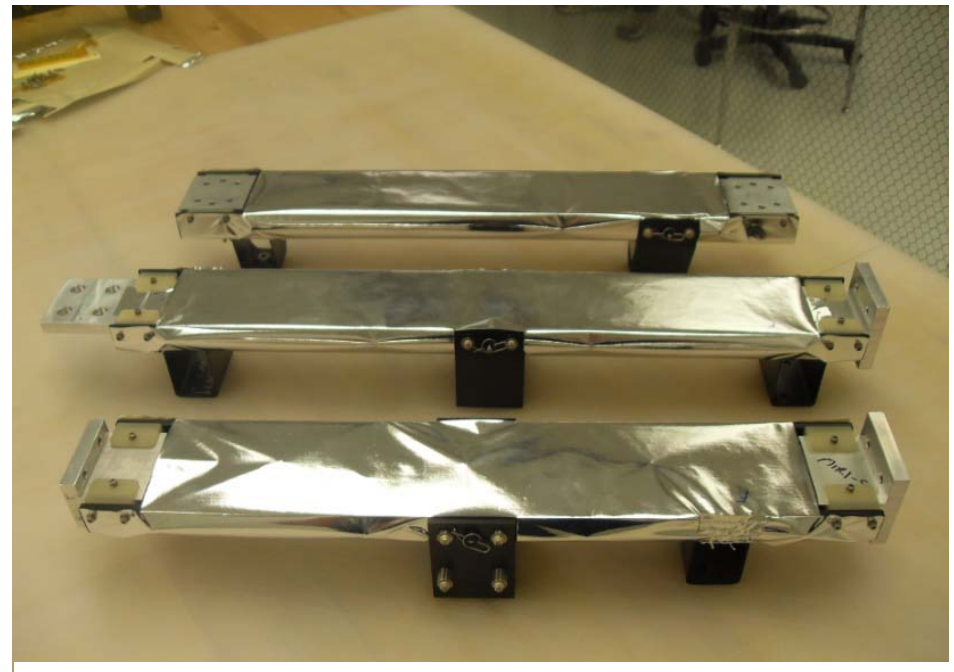

Figure 19: Proto-flight Heat Strap Segments multiple faults to cause significant contamination onto the instruments due to release of entrapped water. A procedure has therefore been developed to decontaminate the instruments should this occur; the same heaters will be used to warm the instruments alone to $165 \mathrm{~K}$, before the structure has time to warm to $140 \mathrm{~K}$. Special control algorithms have been devised to accomplish both the contamination avoidance and decontamination.

Small "trim" heaters provide make-up heat (if needed) to each SI to assure minimum operational temperatures are maintained during science operation. Temperture monitoring throughout the cryogenic ISIM is provided by 93 calibrated CERNOX sensors on the ISIM structure, instruments, radiators, harness radiator, and other surfaces to 
verify that thermal requirements are met, and to be able to monitor ISIM health and safety at all times, including during launch and at other times when science instrument telemitry is unavailable. Figure 20 is a schematic of the ISIM and instrument thermal control scheme.

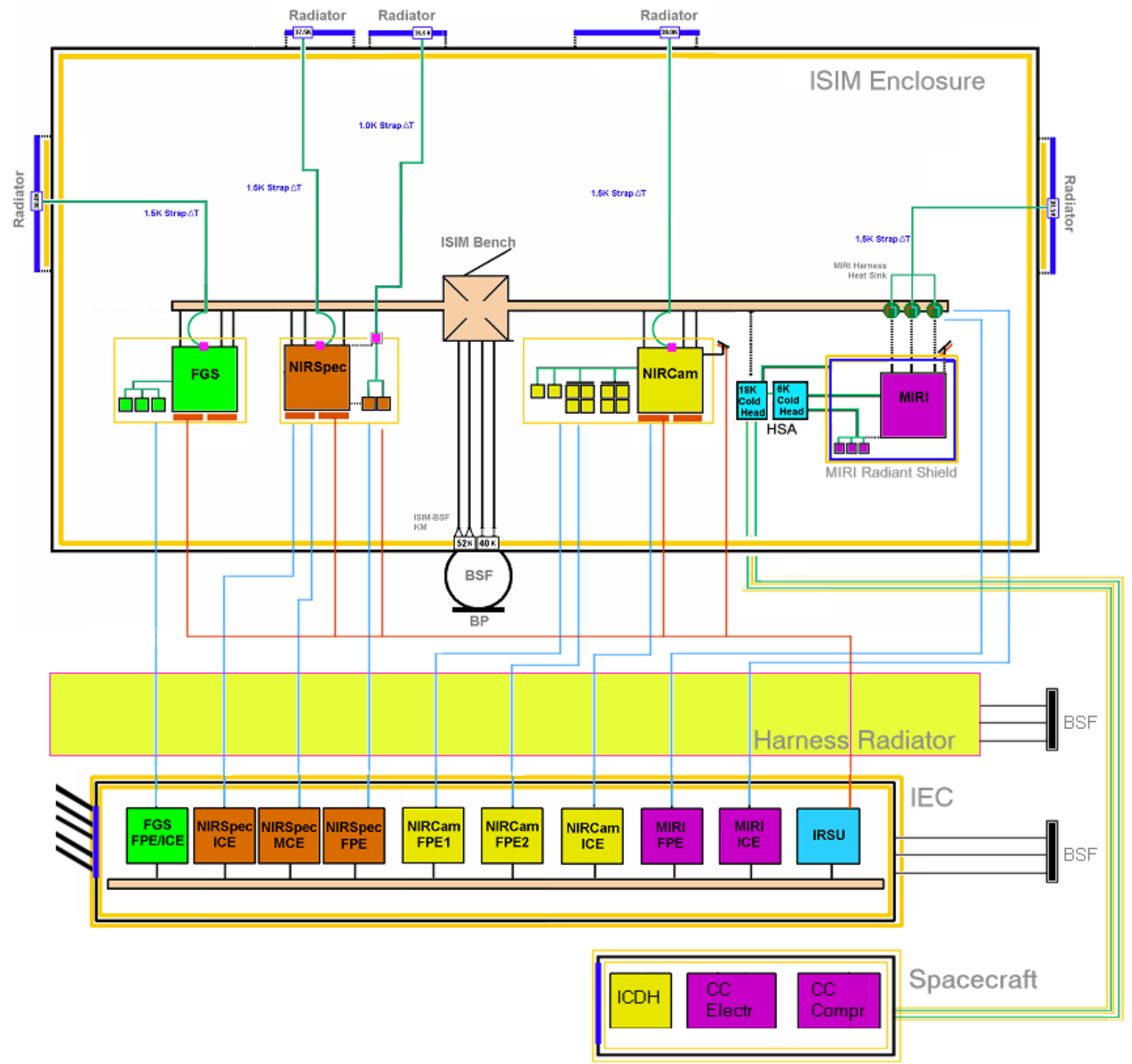

Figure 20: ISIM Thermal Control Schematic

\subsection{Flight Software System}

The ISIM Flight Software (IFSW) is composed of a set of independent tasks that interoperate to process transactions originating from both internal and external sources. The tasks are contained in an operating system environment that is adapted to the mission-specific hardware components with a set of custom device drivers shown in Figure 21 as OS Services. The tasks can be classified into three categories: (1) Command and Telemetry Services, (2) Applications that operate the Science Instruments (SIs) and support science operations, and (3) System Support Services that manage the task environment and external system interfaces.

These tasks have all been developed using IBM ${ }^{\circledR}$ Rational ${ }^{\circledR}$ Rose Real-Time ${ }^{\circledR}(\mathrm{RRT})$, an object-oriented development environment that features visual design of tasks as state machines and definition of inter-task 
messaging as "ports" with "protocols", and which utilizes conventions established in the Unified Modeling Language TM (UML TM). Transitions between states are defined graphically, and behavior code can be associated with transitions or with entry to or exit from a state. Transitions are triggered by specified events such as expiration of a timer or receipt of a signal at a port. The framework code is automatically generated and combined with the behavior code by RRT, which greatly reduces the amount of code that must be written manually. The same development environment was provided to the Science Instrument software development teams to promote ease of integration when their applications are delivered to the ISIM Flight Software Lab at Goddard.

Goddard has defined and implemented, using RRT, a set of standard task interface ports to which every task conforms that allows it to send command packets, receive telemetry packets, receive internal table values for task configuration, receive system time information, perform file operations, use centrally managed timers, and respond to check-in requests to verify that each task is still running. At startup time, each task binds these ports and registers key information that the system needs to provide those services. To date, at least two versions of each SI Application have been integrated with only few and minor problems that were for the most part straightforward to identify and remedy.

To illustrate how the tasks interoperate to provide the desired instrument operation functions, we will describe a few transactions tracing the path taken by successive messages the transaction comprises. Transactions typically originate with the receipt of a command, the expiration of a timer, or the arrival of a telemetry packet. All commands entering the system are messages, sent by a task from its command port, that are queued for service by the Command Manager Task which sends the command to the task indicated by the Application ID in the command packet. A command from the ground will be received by the spacecraft communications system and transferred to the ISIM by the spacecraft Command and Telemetry Processor (CTP). The IFSW CTP Interface Task, which handles the exchange of all commands and telemetry between ISIM and the spacecraft, places the command into a message sent out its command port to the Command Manager Task.

The primary command source in normal operations is the Script Processor Task (SP), which runs scripts written in JavaScript upon receiving a command to do so. The script execution is performed by a JavaScript engine running as separate task that supports multiple concurrent JavaScripts running independently of each other. A set of extensions to the JavaScript language have been implemented that provide the interface to SP, which in turn can access ISIM FSW services through the standard task interface ports. Also, to provide communication between independently running JavaScripts, there are extensions that can set and retrieve the values of shared parameters.

A collection of JavaScripts, stored as ASCII files, make up the Operations Scripts System, discussed in Section 3.9, which provides the capability for automatic operations (see Figure 22). A JavaScript can send a command by communicating to SP, which sends the command packet to the Command Manager. If the command that originated from a JavaScript is an SI function, such as to move a Grating Wheel to a certain position, the command would be routed to the Application Task for that SI. That SI Application Task may generate many commands to the SI hardware to complete the requested operation. These hardware commands are sent via the Command Manager to the bus interface task, either 1553 or SpaceWire, that connects with the SI component being commanded.

The same SI Application Task can send a command to the SI hardware, via the Bus Interface Task, requesting status information to verify that the previous command was properly executed. This hardware status information will be received by the Bus Interface Task and formatted into a Telemetry Packet that is sent out its telemetry port to the Telemetry Manager, which routes the packet to any task that has subscribed to receive packets identified with that Application ID. When the SI Application Task receives that packet, it can decide whether the command succeeded, the operation is complete, or an error has occurred. Every task issues a "housekeeping" telemetry packet at regular intervals providing general status information that can be viewed in real time during a ground contact, or recorded on the Solid-State Recorder and analyzed later on the ground. Also, every task can issue event messages to report errors or successful operations.

The Telemetry Manager Task filters telemetry bound for the SSR and real-time link based on parameters stored in on-board filter tables because the channels that carry telemetry data to the spacecraft CTP have limited capacity. In general, the SI Application Tasks will sustain a higher communication rate through their instrument hardware interface to monitor and control their associated instrument hardware than the rate of commands and telemetry over the user interface through which the SI System is operated, typically by JavaScripts via the Script Processor. 
The Data Acquisition Function is implemented as several tasks that collaborate to marshal science image data through the system to the SSR, and for providing selected image data to other tasks to support boresight guiding and target acquisition. Image data from science observations is never processed by the ISIM Processor. To perform a science data exposure, the user first configures the Data Acquisition Task specifying the entire sequence of images that the exposure is expected to generate. Data Acquisition uses this information to generate a set of frame processing instructions, which it loads into one of the 18 channels provided by the three Focal Plane Array Processor (FPAP) cards. If multiple detectors will be exposed simultaneously, a separated channel is configured for each detector. Once configured, the Data Acquisition Task and the FPAP channels wait for the arrival of science data. Then, the desired SI hardware detector subsystem is configured by sending commands to the SI's Application Task, which in turn sends commands to configure the hardware. When the exposure is commanded to start, data flows into the designated FPAP channel and each frame is processed in accordance with the associated frame processing instruction, which includes information about when to output an image to the SSR. All the image processing is done by the hardware channel so very little CPU time is consumed to capture and record science data. The Data Acquisition Task responds to interrupts and creates header and trailer records for the file sent to the SSR and then triggers the various elements in the science data path to orchestrate the movement of data from the FPAP channels to the SSR. Data Acquisition can also be commanded to retrieve image data subwindows and either write them to files or send them in packets so that the locations of stars can be determined. The FGS System uses this data to send guidance information to the spacecraft CTP to stabilize the telescope by fixing on a guide start. JavaScripts programmed to locate a target star and move it into the desired instrument aperture also use image data.

The remaining software elements perform various monitoring and maintenance functions to keep the software running smoothly, detect problems with the system operation, and restart the software or the processor if uncorrectable problems occur. The Telemetry Monitoring task checks telemetry values against limits stored in a table, and if a value stays outside of the specified limits for a specified length of time, a stored command sequence can be initiated by the Stored Commanding task to take a predefined corrective action. The Memory Scrub task systematically reads all memory locations to detect memory errors caused by radiation using the Error Detection and Correction circuitry in the CPU hardware. The Health and Safety task periodically sends a check-in request to every other task and if a task fails to check in, a software reset is initiated. The Executive Services task can be commanded to send very detailed diagnostic information to ground to assist with analysis of anomalies and the Memory Manager task can update portions of memory to install patches in software to correct minor errors that are discovered.

\subsection{Operations Scripts System}

The Operations Scripts System (OSS) is a collection of JavaScripts (Figure 23) that execute planned science observations and supporting activities, which are specified as a set of ASCII files that are uplinked to the spacecraft from the ground nominally every 10 days. These files are organized around the concept of a visit, which refers to a group of associated science, calibration or engineering activities. A science visit corresponds to a single telescope pointing and includes all the activities that are associated with a single guide star, beginning with the spacecraft slew, and including the guide star acquisition, target acquisition, SI configuration, science exposures, and any small telescope movements needed to fulfill the science objectives.

An Observation Plan, maintained as a file in the ISIM Processor, consists of a list of Visit Records. Each Visit Record contains the acceptable time window for the observation and points to a Visit File that contains lists of activities organized as groups of activity sequences. All the sequences in a group are executed in parallel, and all sequences in a group must complete before advancing to the next group. Groups are processed serially. Observation Plan segments are uploaded and appended to the Observation Plan stored in the ISIM Processor. Visits are executed in the order they appear in the Observation Plan file. After a visit is executed, that Visit Record is deleted from the Observation Plan and the corresponding Visit File is deleted as well. The Activity Sequences within a Visit File consist of activity statements that are executed in order. Each activity statement refers to an activity JavaScript and contains parameters that are passed to the JavaScript. Every type of activity for each SI has a corresponding JavaScript to perform that activity, and parameters in the calling statement are used to control settings that vary from one operation to the next, such as the image window size or which filter wheel to use.

Performing SI operations involves sending commands to the appropriate SI Application task and requesting telemetry back to confirm successful execution of the command. To facilitate the construction of these commands, 
OSS contains a dictionary of all the commands that can be sent that provides the format for each command and its associated parameters. There is a similar dictionary for all the telemetry values that can be requested that specifies the telemetry packet ID and offset where that parameter can be found in the packet. There are interface JavaScripts that provide the access to this information to the JavaScripts that are performing these command and telemetry operations.

The reading, interpretation and execution of the Observation Plan and the Visit Files are conducted by a set of JavaScripts, collectively called the Observation Plan Executive (OPE), that run under the control of the Script Processor Task (SP). SP can run up to ten independent JavaScript threads each in a separate "context". This allows the OPE to simultaneously manage the Observation Plan and the Visit File execution, to manage multiple concurrent visit file activities, to interact with the spacecraft to coordinate reaction wheel momentum unloading, and to respond to requests from the ground to start, stop or modify the Observation Plan. SP provides the interface between the JavaScripts and the ISIM FSW services described in Section 3.8, which include sending and receiving commands and telemetry and reading and deleting files. A command from the ground to SP to run the top-level OPE script starts the automated observing process, which continues as long as there are visits to process, until a command is sent from the ground to stop or suspend automatic observing, or until an error occurs that requires ground attention.

SP carefully monitors and regulates resources for JavaScript execution within each context including memory allocation and release, CPU execution time slicing, and limiting the frequency that telemetry packets are issued. Limiting CPU execution time is necessary because some scripts, such as the ones that perform target acquisition, can be very CPU intensive and can run for a long time. Limiting how long each context can use the CPU ensures that tasks with real-time deadlines always have enough time to perform their functions.

The fact that the OSS is written in JavaScript and stored on-board as ASCII files is significant because this gives the operations personnel greater visibility, control and flexibility over the telescope operations. As they learn the ramifications and subtleties of operating the instruments, they can modify the JavaScripts and, after thorough testing in a ground facility, they can simply replace an onboard file to make the change. The JavaScripts are written using the terminology of the user's domain of commands and telemetry. This is in contrast to the ISIM FSW tasks, which involve real-time state machine logic, intricate hardware interaction, intertask message routing, a hierarchy of task priorities, and complex operating system interfaces. The OSS is in the user domain and is consistent with the users' perspective, needs and objectives. The ISIM FSW is in the system domain and provides the user with access to the functions and features of the system without the need for detailed knowledge of the underlying implementation.

Another significant feature of the OSS, a feature that pervades the end-to-end science operations philosophy of JWST, is the notion of event-driven operation. In the JSWT context, event-driven operation means simply that the sequence of operations is specified, and that the next step is performed when the previous step completes. Earlier generations of spacecraft relied on an uplinked command sequence stored in "command memory" with every command specified down to the bit with an associated time tag. The on-board computer would simply read each command, and would, at the designated time, send the command to the intended piece of hardware. This meant that every detail of spacecraft and science operations had to be modeled on the ground so that accurate calculations of the command execution times could be performed and that worst-case times for every operation had to be assumed. If an operation failed, for example, because a guide star was not acquired, operations stopped. At the next real-time contact, the ground staff would discover the problem and engage in a flurry of activity to create a new command load, which would be uplinked at a subsequent real-time contact. As flight processors became faster and radiationhardened memory increased in capacity, on-board automation evolved as well. With event-driven operations, if a guide star acquisition fails, that visit is skipped and the OPE simply advances to the next visit, which greatly increases the observing efficiency of the telescope and greatly simplifies the ground system as well.

For further detail on the OSS, see paper 7737 of this conference. 


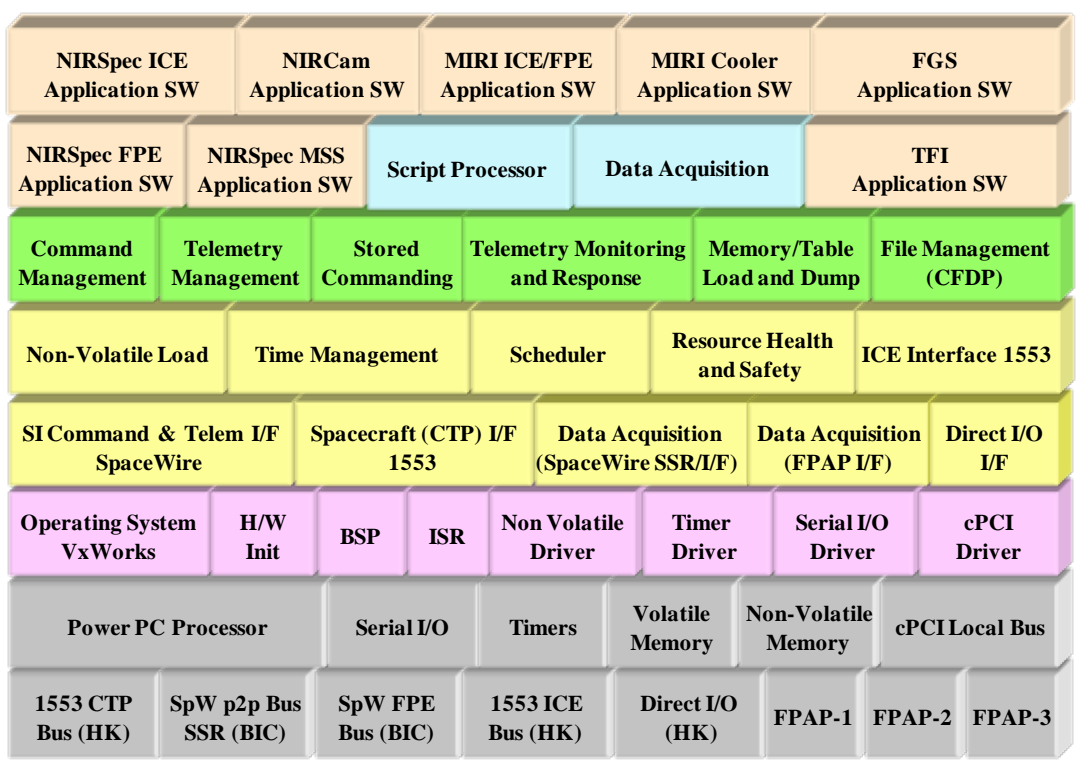

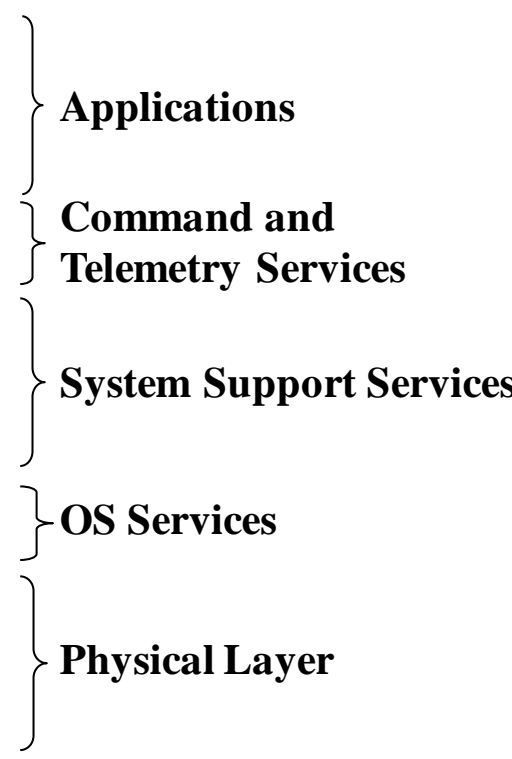

Figure 21. ISIM Flight Software Layered Task Architecture

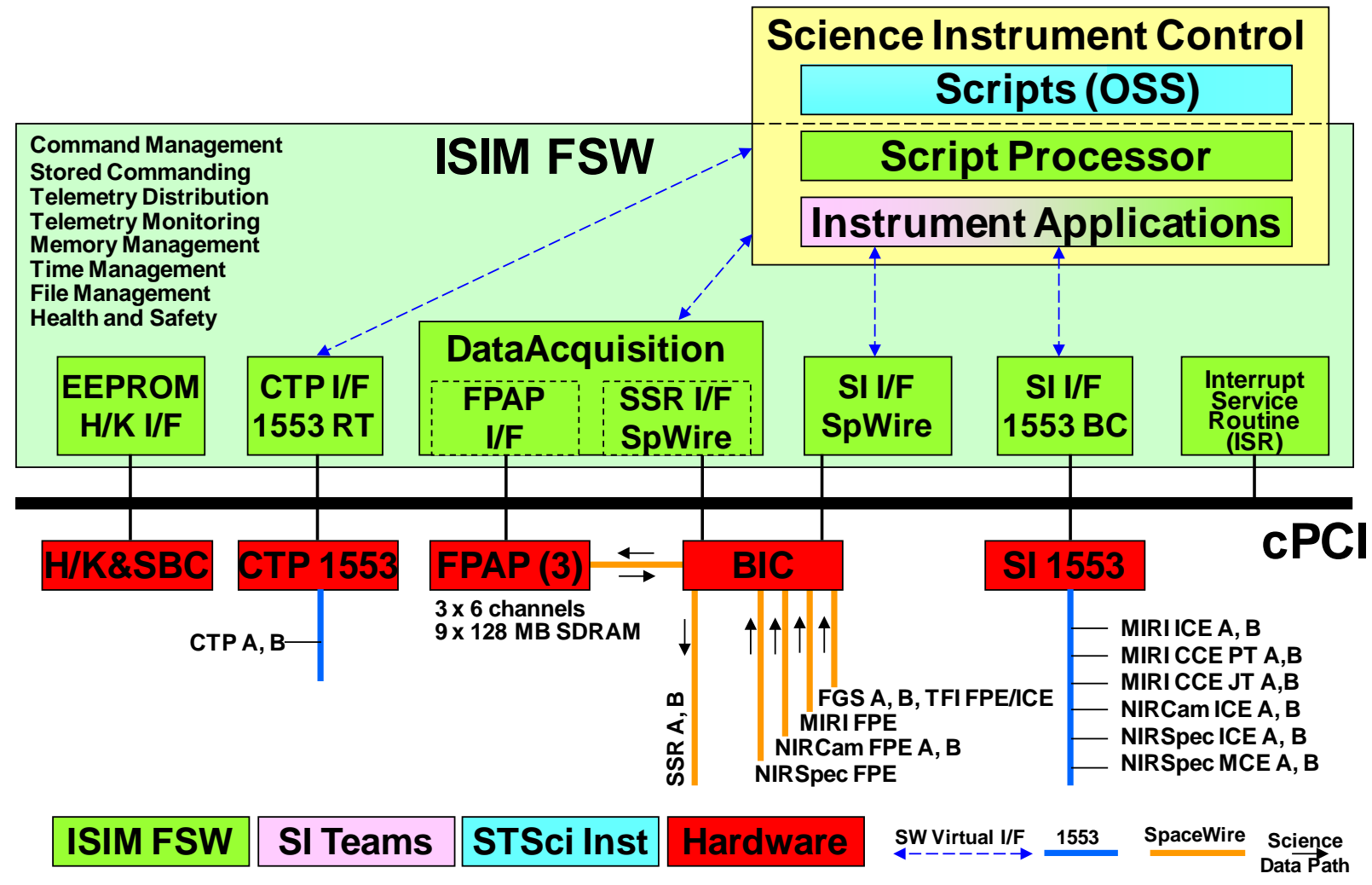

Figure 22. ISIM Flight Software Environment from an Operational Perspective 


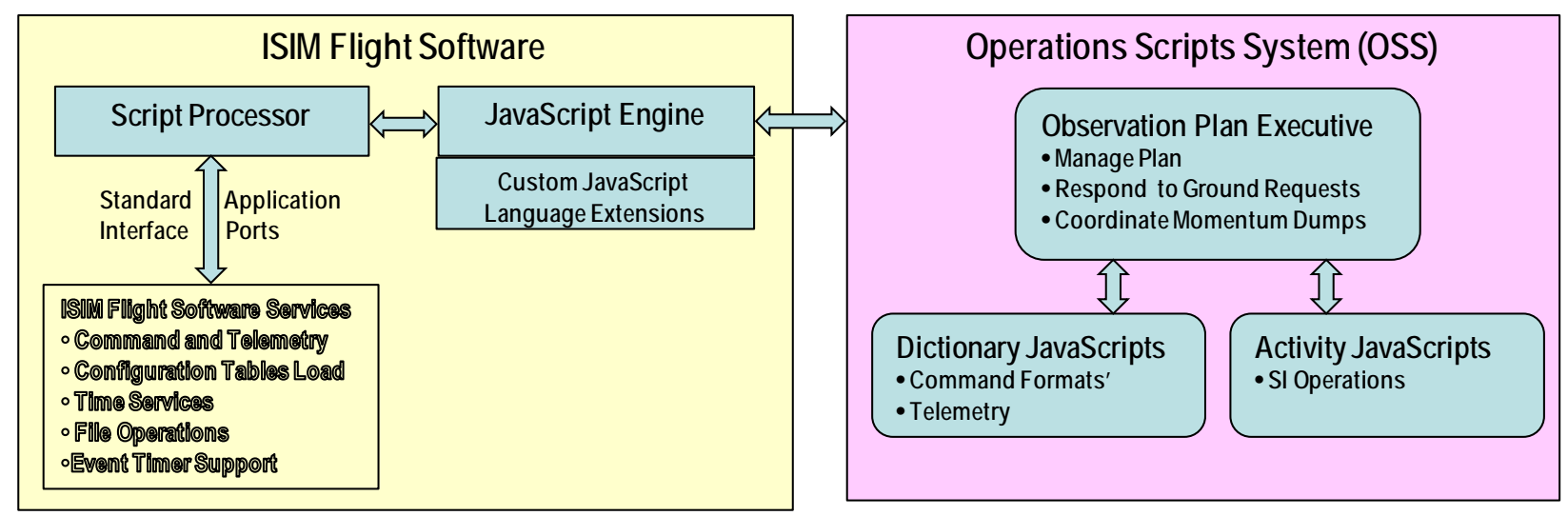

Figure 23. OSS JavaScripts Communicate to the ISIM FSW Using Custom Language Extensions

\section{OPTICAL INTEGRATION AND TEST}

The major subsystems that make up the ISIM Element are separately verified before delivery for Element-level integration and test (I\&T), during which the Element is verified to meet its requirements after assembly: The SI developer verifies that the SI meets its optical performance and optomechanical alignment requirements as a standalone unit, assuming nominal Optical Telescope Element (OTE) performance. The ISIM structure is verified to meet its mechanical alignment requirements, and it is optically tested after integration to verify that the whole ISIM meets its performance requirements after integration of the instruments with their supporting systems (Sec 3) and exposure to simulated launch and operational environments.

\subsection{Integration and alignment}

For ISIM Element development, the JWST optomechanical coordinate system for the science payload, the "vehicle coordinate system" (VCS), is represented on interface tooling. ${ }^{10,11}$ The SIs are optically aligned to their mechanical interface to the ISIM structure using this tooling (Figure 24a). The ISIM Element is aligned to its interface to the OTE using another precision fixture, the ISIM Test Platform (ITP) (Figure 24b and 25a).

The alignment fixture used for SI alignment and verification to the ISIM structure is called the ambient science instrument mechanical interface fixture (ASMIF). It is a metal platform about the size of the SI footprint on the ISIM structure. When the SI is mounted to its ASMIF, the ASMIF supports the full SI load. The type of metal and dimensions vary from SI-to-SI, because each interface is mechanically different and each SI has a different mechanical design. The ASMIF has a flight-like, precision representation of the SI-ISIM interface (i.e., tapped holes, precision pin holes and slots for clocking pins). The ASMIF is only used at ambient. The SI developer relates the cryogenic alignment of the SI to this ambient fixture. The ASMIF is thus a "master gauge" for SI-ISIM alignment.

The ITP is the alignment fixture used for ISIM alignment and verification relative to its interface to the OTE. The ITP is an invar platform that holds the ISIM structure and SIs and, thus, has a footprint of $\sim 2.2 \times 2.2 \mathrm{~m}$ and contains OTE-like interface features. The ITP is a bolted and pinned, invar structure that is stress relieved for optimum stability and repeatability at ambient and cryogenic conditions. The difference between the ITP and a nominal telescope backplane is an important factor in ground alignment adjustment prior to delivery of ISIM to OTE for final integration. 
The SI interface on the ISIM structure is expected to change very little (i.e., $~<0.6 \mathrm{~mm}$ in translations) as a result of temperature change from ambient to the cryogenic operating temperature and all other ground-to-orbit effects (e.g., moisture desorption, g-release uncertainty). The various sources of ground-to-orbit change have been identified and estimated based on extensive modeling validated by component and assembly test articles. The levels of change are small compared to ISIM top-level performance requirements. We are therefore able to treat excursions from nominal in the SI-ISIM interface as error budget terms.

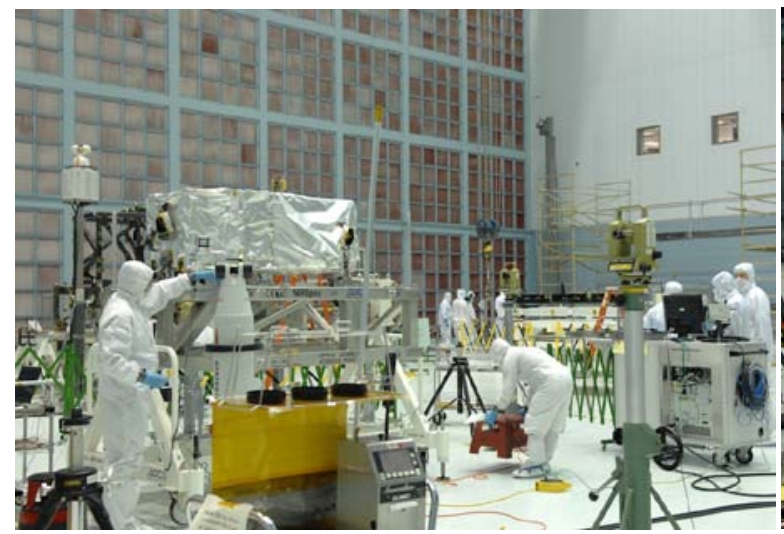

(a)

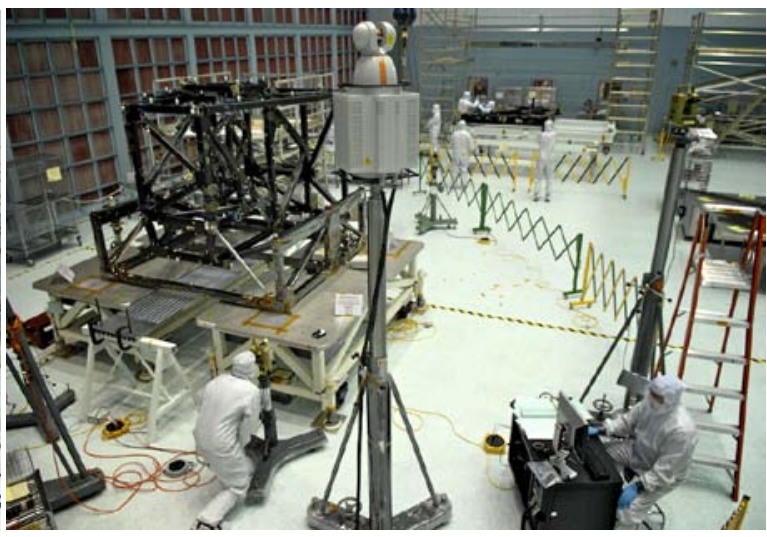

(b)

Figure 24: a. The ETU NIRSpec instrument (middle, left) during post-shipment metrology operations. b. Foreground: The ISIM Structure (left) undergoing coordinate system metrology using LIDAR and theodolite instruments. Background: The ITP is being readied for cryogenic testing.

Commercial laser tracker, LIDAR, and theodolite instruments are used for ambient metrology and envelope measurement. ${ }^{12}$ Photogrammetry (PG) is used for cryogenic metrology of the ITP and ISIM structure (Figure 25b) ${ }^{13}$ Cryogenic characterization of the ITP was completed in early 2010. The ITP dimensional change from ambient to cryogenic temperatures is in good agreement with model expectations. The structure cryogenic alignment was measured in spring 2010, with good results. The custom, cryogenic PG system performed better than its required measurement uncertainty.

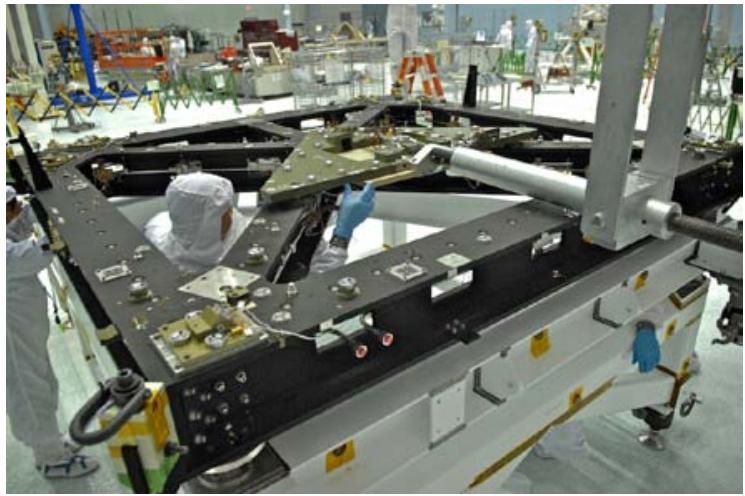

(a)

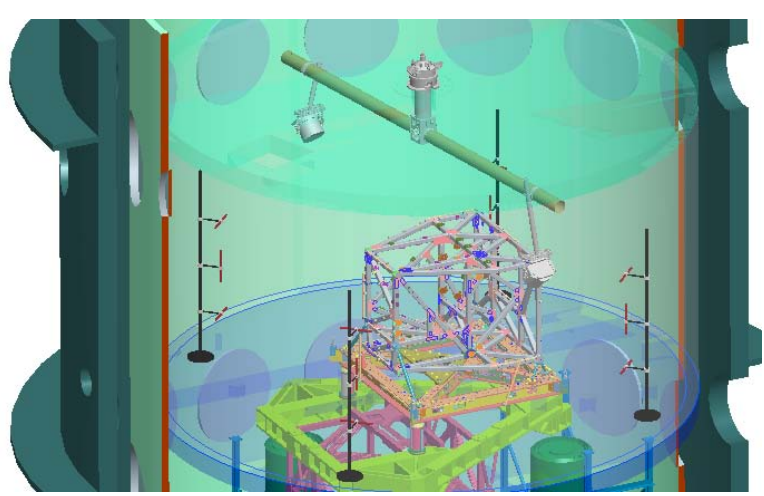

(b)

Figure 25: a. The ITP surrogate telescope backplane, showing integration of the MATF fixture in preparation for cryogenic metrology. b. CAD rendering of the Flight ISIM structure and cryogenic alignment performance verification via $P G$ in the SES chamber. 


\subsection{Optical testing and verification}

Before integrating the ISIM with the OTE, the ISIM is verified to meet its requirements relative to its interface. Furthermore, the performance of the SIs is calibrated at the ISIM-level in order to support later OTE-level ground testing and on-orbit, Observatory-level commissioning. This battery of optical test and verification is accomplished using a full-field, cryogenic optical stimulus, the OTE SIMulator (OSIM) developed by GSFC and Ball Aerospace (Figure 26). ${ }^{14}$

For the optical verification or "end-to-end" test of the ISIM element, the OSIM is used to simulate a star field generated by a nominal OTE, to within limits of alignment uncertainty, fabrication of the OSIM optics, design residual, etc. In order to meet OSIM-to-nominal-telescope-interface alignment requirements, OSIM must calibrate its output relative to this mechanical interface on the ITP. This calibration is performed during a series of optical tests prior to testing ISIM with OSIM.

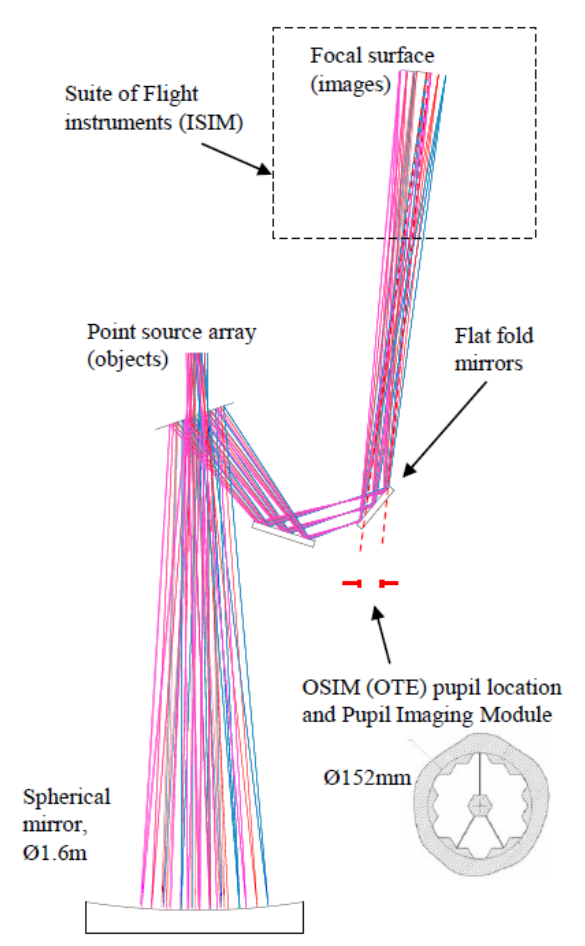

(a)

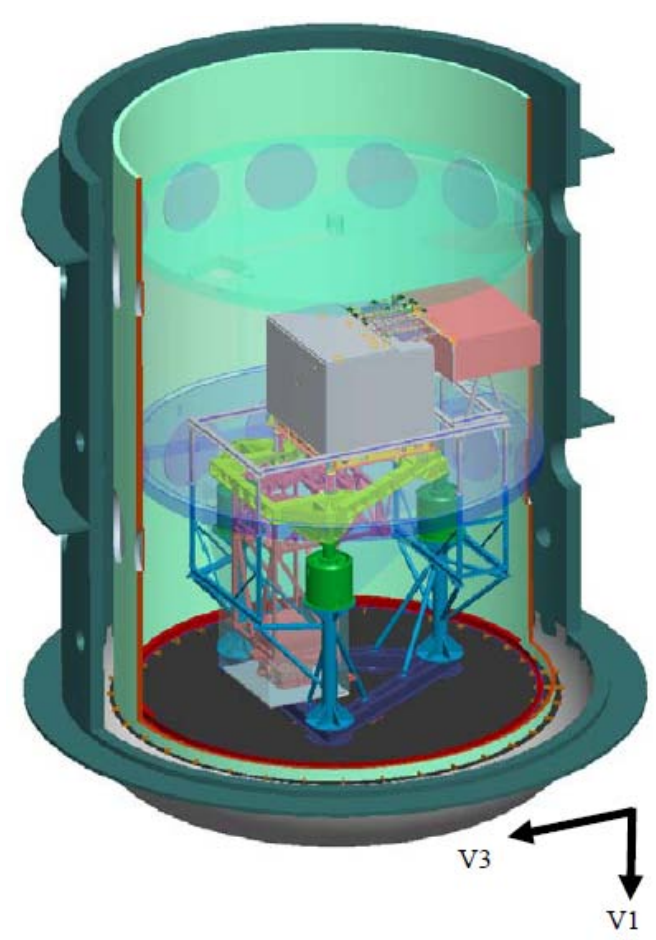

(b)

Figure 26: a. Ray trace schematic of the OSIM optical layout. b. CAD image of the ISIM Element and cryogenic performance verification using OSIM in the SES chamber.

During the calibration of OSIM, PG measures the location and orientation of the Master Alignment Target Fixture (MATF) (Figure 25a). The MATF is an invar plate that is pinned to the ITP, and allows OSIM to align its optical output to the mechanical OTE-ISIM interface. The MATF hosts an array of PG targets on its surface that are used for measuring MATF six degree-of-freedom alignment relative to the ITP. The MATF is also equipped with an array of five optomechanical targets that are not visible to the PG system, but are designed to provide an optomechanical reference for OSIM's in situ alignment.

The optical verification of the ISIM Element using OSIM is divided into multiple sub-test categories: SI basic functionality and internal calibration; focus; pupil shear; boresight and field of view; vignetting and stray light; wavefront error; co-boresight stability; wavefront sensing. The first category of tests is performed to establish the functionality of the SI mechanisms, detectors, calibration lamps, etc. --- this battery of checks will performed periodically to trend behavior after certain I\&T evolutions and environmental exposure. The tests related to optical alignment (i.e., focus, pupil shear, boresight) are associated with ISIM Element requirements for SI alignment as an assembly of instruments. SI alignment relative to each other and, as a suite, relative to the ISIM-OTE interface is 
verified via this test, with significant analysis to account for ground effects. The co-boresight stability test is a thermal over-drive test, where the relative alignment of the SIs is monitored as a function of temperature. The SI wavefront error (i.e., image quality) is measured using phase retrieval techniques. The as-built wavefront error is calibrated for future reference during verification activities at the Observatory-level and on-orbit alignment of the telescope. Also in support of on-orbit telescope assembly and commissioning, the ability to perform wavefront sensing-related measurements is also verified. 


\section{REFERENCES}

1. Gardner, Jonathan P., "Science with the James Webb Space Telescope”, Proc. SPIE Int. Soc. Opt. Eng., [7731-05], 2010.

2. Greenhouse, Matthew A., et al., "The James Webb Space Telescope; Mission Overview and Status”, in Science with the 8-10m telescopes in the era of the ELTs and the JWST, The Ramon Areces Foundation, p. 168, 2009.

3. Gardner, Jonathan P., et al., "The James Webb Space Telescope”, Space Science Reviews, 123: 485, 2006.

4. Greenhouse, Matthew A., et al., "The James Webb Space Telescope Integrated Science Instrument Module”, Proceedings of the SPIE, Vol. 6265, p. 626513, 2006

5. Greenhouse, Matthew A., et al., "The James Webb Space Telescope Integrated Science Instrument Module”, Proceedings of the SPIE, Vol. 5487, p. 754, 2004

6. Greenhouse, Matthew A., et al., "The NGST Integrated Science Instrument Module”, Proceedings of the SPIE, Vol. 4013, p. 795, 2000

7. Rieke, M. 2005, ” Overview of James Webb Space Telescope and NIRCam's Role,” Proc. SPIE, 5904, 1

8. Rieke, M. 2008, see: http://ircamera.as.arizona.edu/nircam/AAS_June08.pdf

9. Wright et al. 2008, "Design and development of MIRI, the mid-IR instrument for JWST," Proc. SPIE, 7010, 27

10. Hagopian, J., Ohl, R., Bos, B., Davila, P., Eichhorn, W., Hylan, J., Hill, M., Nowak, M., Pasquale, B., Sampler, H., Wilson, M., Gallagher, B., Hardaway, J., Sullivan, J., Young, P., Keepers, T., and Quigley, R., "Optical alignment and test of the James Webb Space Telescope Integrated Science Instrument Module," Integration, Alignment and Test of Large Optical Systems, IEE Aerospace Conf., 2007.

11. Ohl, R., "Updates to the optical alignment and test plan for the James Webb Space Telescope integrated science instrument module," Proc. SPIE 7433, 743305, 2009.

12. Ohl, R., Eegholm, B., Casas, M., Frey, B., Dominguez, M., Gill, J., Hayden, J., Morken, P., Redman, K., Roberts, V., Saif, B., and Scirpo, T., "Trades for Ambient Non-Contact Metrology," Proc. SPIE 7433, 743306, 2009.

13. Nowak, M., Cleveland, P., Crane, A., Davila, P., Herrera, A., Hylan, J., Liehr, A., Marsh, J., Ohl, R., Redman, K., Sampler, H., Stock, J., Wenzel, G., Woodruff, R., and Young, P., "Verification of the James Webb Space Telescope Integrated Science Instrument Module Cryogenic Structural Alignment Requirements via Photogrammetry,” Proc. SPIE 7068, 70680Q, 2008.

14. Davila, P., Bos., B., Cheng, E., Chang, B., Eichhorn, W., Frey, B., Garza, M., Gong, Q., Greeley, B., Guzek, J., Hakun, C., Hovmand, L., Kirk, J., Kubalak, D., Leviton, D., Nagle, A., Nyquist, R., Pham, T., Robinson, F. D., Sabatke, D., Sullivan, J., Volmer, P., von Handorf, R., and Youngworth, R., "Optical Telescope Element Simulator for the James Webb Space Telescope,” Proc. SPIE 7010, 70103F, 2008. 